\title{
Preventive effects of Polygonum multiflorum on glucocorticoid-induced osteoporosis in rats
}

\author{
MANRU ZHOU ${ }^{1,2}$, JIN LI $^{1}$, JINGKAI WU ${ }^{1}$, YAJUN YANG ${ }^{1}$, XIAOBING ZENG ${ }^{3}$, \\ XIAOHUA LV ${ }^{1}$, LIAO CUI ${ }^{1,3}$, WEIMIN YAO ${ }^{4}$ and YUYU LIU ${ }^{1}$ \\ ${ }^{1}$ Department of Pharmacology, Guangdong Medical University, Zhanjiang, Guangdong 524023; \\ ${ }^{2}$ Department of Pharmacy, Xinhua College of Sun Yat-sen University, Guangzhou, Guangdong 510520; \\ ${ }^{3}$ Guangdong Key Laboratory for Research and Development of Natural Drugs; ${ }^{4}$ Department of Respiratory Medicine, \\ Affiliated Hospital of Guangdong Medical University, Zhanjiang, Guangdong 524023, P.R. China
}

Received March 28, 2016; Accepted March 10, 2017

DOI: $10.3892 /$ etm.2017.4802

\begin{abstract}
In Traditional Chinese Medicine, Polygonum multiflorum (PM) is known for its anti-aging properties. A previous study by our group showed that extracts of PM were able to prevent and treat bone loss in vivo, and the active components emodin and 2,3,5,4,-tetrahydroxystilbene-2- $O-\beta$ -glucoside (TSG) promoted the osteogenic differentiation of mesenchymal stem cells in vitro. The aim of the present study was to investigate the preventive effects of PM on glucocorticoid-induced osteoporosis (GIO) in rats. A crude extract of PM was prepared with $75 \%$ ethanol, purified and enriched using a D-101 macroresin column and elution with $30 \%$ ethanol, and the material obtained was assessed by high-performance liquid chromatography. Male or female Sprague Dawley rats $(\mathrm{n}=180)$ were randomly divided into nine groups: Control, prednisone, prednisone plus calcitriol (CAL), prednisone plus $30 \%$ ethanolic eluate of PM [high (H), medium (M) and low (L) dose] and prednisone plus crude extract of PM (H, M and L dose). Prednisone was orally administered to the osteoporosis model rats for 21 weeks, alongside which they received PM extracts. The weight of the viscera, anterior tibial muscle and other tissues was recorded at the end of the experiment. The femur and lumbar vertebra were collected for the measurement of three-dimensional microarchitecture by micro-computed tomography scanning, assessment of biomechanical properties and determination of bone mineral density (BMD). In the $30 \%$
\end{abstract}

Correspondence to: Professor Yuyu Liu, Department of Pharmacology, Guangdong Medical University, 2 Wenmingdong Road, Zhanjiang, Guangdong 524023, P.R. China

E-mail: liuyuyu77@163.com

Dr Weimin Yao, Department of Respiratory Medicine, Affiliated Hospital of Guangdong Medical University, 57 Renmindadaonan Road, Zhanjiang, Guangdong 524023, P.R. China

E-mail: 490296443@qq.com

Key words: Polygonum multiflorum, prednisone, osteoporosis, rats, prevention ethanolic eluate of the PM extract, the content of TSG and combined anthraquinone was 9.20 and $0.15 \%$, respectively, and that in the crude extract of PM was 2.23 and $0.03 \%$, respectively. Over 6 weeks, the weight of the rats the in prednisone group decreased $(\mathrm{P}<0.05)$, while the weight of rats treated with $\mathrm{M}$ and $\mathrm{H}$ doses of $30 \%$ ethanolic eluate was increased compared with that in the prednisone group $(\mathrm{P}<0.05)$. Rats exposed to prednisone exhibited a deteriorated bone microarchitecture, low BMD, decreased bone volume/total volume and poor biomechanical properties. Furthermore, the weight of the adrenal gland and the anterior tibial muscle was decreased. $30 \%$ ethanolic eluate of PM at M and L doses and crude extract of $\mathrm{PM}$ at the $\mathrm{H}$ dose counteracted the alterations of skeletal and other characteristics induced by prednisone in rats, as did CAL. In conclusion, extracts of PM exerted a protective effect on bone tissue in GIO rats.

\section{Introduction}

Glucocorticoids (GCs) are widely used in chronic inflammatory and autoimmune diseases (1-4). However, long-term excessive administration of GCs elicits significant bone loss $(5,6)$ resulting in severe fracture of various bones in $30-50 \%$ of patients (7-10). GC-induced osteoporosis (GIO) is one of side effects of GCs, which contributes to an increase of morbidity and mortality (11). Measures of prevention and treatment of GIO should be taken for patients receiving GCs (8). While this deleterious effect of GCs has become increasingly known over previous decades, GIO remains under-treated in patients receiving GCs (12). Patients exposed to GCs for a long period of time ( $\geq 6$ months) should take anti-osteoporotic drugs in order to prevent GIO (3). In addition, the major cause of GIO is considered to be impairment of bone formation $(13,14)$. Although parathyroid hormone as an approved anabolic agent is thought to be a beneficial therapy for the prevention and/or treatment of GIO, its clinical usage is limited due to its high cost (15). Therefore, there is an urgent requirement for the development of medications for the prevention and treatment of GIO (16-18).

The dried roots of Polygonum multiflorum Thunb (PM), a medicinal herb, have been widely used in Traditional Chinese 
Medicine for thousands of years. A previous study by our group showed that extracts of PM prevented and recovered bone loss in ovariectomized rats (19), which has been patented (20). Further studies by our group revealed the effects of the major active components of PM, such as emodin and 2,3,5,4'-tetrahy droxystilbene-2- $O$ - $\beta$-glucoside (TSG), on osteogenic differentiation and bone formation $(21,22)$. Subsequent studies by our group showed that emodin and TSG promote the osteogenic differentiation of mesenchymal stem cells and the underlying mechanism involves the regulation of the Wnt signaling pathway $(23,24)$. Whether PM has a preventive effect on GIO has remained elusive. Based on the abovementioned studies, the present study aimed to investigate the content of TSG (\%) and combined anthraquinone (CAQ) (\%) in various extracts of $\mathrm{PM}$, and to assess the effects of PM on bone mass, architecture and biomechanical properties in GIO rats.

\section{Materials and methods}

Preparation of the extracts. PM was purchased from Yulin Xiang Sheng Chinese Herbal Medicine Co., Ltd. (Nanyang, China) and characterised by Professor Yuyu Liu at the Department of Pharmacology of Guangdong Medical University (Zhanjiang, China). A voucher specimen was deposited at the herbarium of Guangdong Key Laboratory for Research and Development of Natural Drugs, Guangdong Medical University (Zhanjiang, China). The plant material was air-dried indoor at room temperature. Air-dried roots of PM $(56.0 \mathrm{~kg})$ were extracted with $75 \%$ ethanol at $55^{\circ} \mathrm{C}$ for $4 \mathrm{~h}$, followed by rinsing with cyclohexane. A crude extract of the PM $(9.25 \mathrm{~kg})$ was acquired by evaporation of the organic solvent under reduced pressure at $55^{\circ} \mathrm{C}$. The PM extract $(2 \mathrm{~kg})$ was dissolved in water and loaded onto a D-101 macroresin (Shaanxi Lanshen Special Resin Co., Ltd., Xian, China) column (80x1,200 mm), and then successively eluted with $\mathrm{H}_{2} \mathrm{O}$ as well as $10,20,30,40,50,60$, 70,80 and $90 \%$ ethanol. According to preliminary results on the bioactivity of the extracts, the $30 \%$ ethanolic eluate of PM showed the highest potency in promoting the differentiation of cultured osteoblasts.

Preparation of standard and sample solutions for high-performance liquid chromatography (HPLC) examination. Reference compounds of TSG, emodin and physcion were purchased from the National Institute for Food and Drug Control of China (batch nos. 110844-201411, 110756-200110 and 110758-201415, respectively). The three reference compounds were accurately weighed and then dissolved in $70 \%$ methanol to produce standard solutions. Each powdered sample of $0.5 \mathrm{~g}$ was refluxed using $50 \mathrm{ml} 70 \%$ methanol in a water bath for $60 \mathrm{~min}$. The supernatant was used as test solution A for TSG and free anthraquinone (FAQ). Test solution A $(10 \mathrm{ml})$ together with $1.5 \mathrm{ml} 36 \%$ hydrochloric acid was refluxed in a water bath for $60 \mathrm{~min}$ and then transferred to a $25-\mathrm{ml}$ volumetric flask, diluted with $70 \%$ methanol to volume and mixed well. The supernatant of this prepared solution was used as test solution B to determine total anthraquinone (TAQ) content. The CAQ content was calculated as follows: Content of CAQ=content of TAQ-content of FAQ (25). CAQ was calculated as the total amount of physcion and emodin. Standard sample and test solutions A and B were filtered through $0.45-\mu \mathrm{m}$ membranes separately, and $10 \mu \mathrm{l}$ of each standard and sample solution was analyzed by HPLC 6 times. The contents of TSG and CAQ in the PM extracts were measured by HPLC on a Syncronis C18 column (250x4.6 mm) on an Agilent 1200 HPLC system (Agilent Technologies, Santa Clara, CA, USA). The data were obtained under the following HPLC conditions: Flow rate, $1.00 \mathrm{ml} / \mathrm{min}$; injection volume, $10 \mu \mathrm{l}$; column temperature, $25^{\circ} \mathrm{C}$; mobile phase $\mathrm{A}, \mathrm{MeOH}$ and $\mathrm{B}, \mathrm{H}_{2} \mathrm{O}$ with $0.1 \%$ phosphoric acid; elution program: Linear gradient from 10-30\% A over $20 \mathrm{~min}$, to $50 \%$ A over $20 \mathrm{~min}$, to $85 \%$ A over $20 \mathrm{~min}$, to $100 \%$ A over $20 \mathrm{~min}$, which was then maintained for $20 \mathrm{~min}$; detection wavelength, $320 \mathrm{~nm}$ for TSG and $254 \mathrm{~nm}$ for physcion and emodin.

Animal experiment. The study was approved by the Academic Committee on the Ethics of Animal Experiments of Guangdong Medical College [Zhanjiang, China; permit no. SCXK (Guangdong) 2008-0008]. All animals were treated in accordance with the Guidelines and Regulations for the Use and Care of Laboratory Animals of Guangdong Laboratory Animal Monitoring Institute under the National Laboratory Animal Monitoring Institute of China. Male and female Sprague Dawley rats (age, 6 months; weight, 190-230 $\mathrm{g} ; \mathrm{n}=180$ ) were randomly divided into eighteen cages at the Animal Center of Guangdong Medical University (Zhanjiang, China) and acclimatized for two weeks at $24-28^{\circ} \mathrm{C}$ and $50-60 \%$ humidity. At the beginning of the study the weight of the female rats was $200 \pm 20 \mathrm{~g}$, and the weight of the male rats was $220 \pm 20 \mathrm{~g}$. The rats were randomly divided into nine groups: Control (normal saline), prednisone (6 $\mathrm{mg} / \mathrm{kg} / \mathrm{day})$, prednisone ( $6 \mathrm{mg} / \mathrm{kg} / \mathrm{day}$ ) plus calcitriol (CAL; $0.045 \mu \mathrm{g} / \mathrm{kg} /$ day), prednisone $(6 \mathrm{mg} / \mathrm{kg} / \mathrm{day})$ plus $30 \%$ ethanolic eluate of PM at a high $(\mathrm{H})$, medium (M) or low (L) dose (400, 200 or $100 \mathrm{mg} / \mathrm{kg} /$ day, respectively) or prednisone $(6 \mathrm{mg} / \mathrm{kg} /$ day) plus crude extract of PM at a high (H), medium (M) or low (L) dose $(400,200$ or $100 \mathrm{mg} / \mathrm{kg} /$ day, respectively). Rats were administered prednisone for 21 weeks per oral gavage as a GIO model. Furthermore, the extracts mentioned above were administered to rats by oral gavage exposed to prednisone, Prednisone was administered in the afternoon, while the extracts were administered in the morning. Changes in body weight were monitored every week. The weight of the viscera, anterior tibial muscle and other tissues were recorded at the end of the experiment (21 weeks). The femur and lumbar vertebrae (LV) were collected for the measurement of the three-dimensional (3D) microarchitecture via micro-computed tomography (micro-CT) scanning as well as determination of biomechanical properties and bone mineral density (BMD) (26).

Micro-CT examination. The right femur and the fourth $\mathrm{LV}$ $\left(\mathrm{LV}_{4}\right)$ were dissected from the residual muscle and secured in a sample holder, and micro-CT imaging was performed on a SCANCO VivaCT 40 (Scanco Medical, Zurich, Switzerland) (27). Samples were acquired with 1,000 projections for 2,048 samples each, reconstruction of 2,048x2,048 pixels, integration time 8 of $200 \mathrm{msec}$, an energy of $70 \mathrm{kVp}$, a current of $114 \mathrm{~mA}$ and a diameter of $38.9 \mathrm{~mm}$. Each measurement included 530 slices corresponding to a $9.50-\mathrm{mm}$ thick cross-section of the forearm. The values for the lower and upper threshold were set as 170/1,000 and 220/1,000 in male 
and female rats, respectively. The regions of interest of femoral cancellous were selected for analysis. Using the cross-sectional images from micro-CT scanning, longitudinal analysis of the distal femur excluding the cortical bone was performed in the measurement area between 1 and $4 \mathrm{~mm}$ distal to the growth plate-metaphysis junction (28-30). As for the $\mathrm{LV}_{4}$, the trabecular bone region was outlined for each micro-CT slice, excluding the cranial and caudal endplate regions at $1 \mathrm{~mm}$ from the growth plate-metaphysis junction (31). 3D images were obtained for visualization. Bone volume/total volume (BV/TV), structure model index (SMI), trabecular number (Tb.N), trabecular separation (Tb.Sp), trabecular thickness (Tb.Th), H-h, B-b, BMD and density at the bone tissuses were measured (Fig. 1). All of the micro-CT analyses were performed using the Scanco micro-CT software analyzing system (Scanco Medical; v. 2013).

Assessment of BMD. The right femur and the $\mathrm{LV}_{4}$ were removed and the residual muscle was dissected. For maintaining moisture, samples were wrapped with saline-saturated gauze and stored at $-20^{\circ} \mathrm{C}(32)$. BMD was measured by dual-energy X-ray absorptiometry (Discovery Wi; Hologic, Inc., Bedford, MA, USA).

Determination of biomechanical properties. The right femurs were obtained and their bone mechanical properties were determined through three-point bending (33). The upper and lower sides of the cartilage of the $\mathrm{LV}_{5}$ were rubbed off and the $\mathrm{LV}_{5}$ was used to measure the bone mechanical properties through compressing. An MTS Mini Bionix testing system (Mini Bionix858, MTS, USA) was used for the measurements (32). Samples were tested with a $15-\mathrm{mm}$ span at a speed of $0.01 \mathrm{~mm} / \mathrm{sec}$. Load-displacement curves were plotted. The output parameters included elastic load $(\mathrm{N})$, maximum load $(\mathrm{N})$, break load $(\mathrm{N})$ and stiffness $(\mathrm{N} / \mathrm{mm})$.

Statistical analysis. Values are expressed as the mean \pm standard deviation. Statistical analyses were performed with SPSS version 22.0 (IBM SPSS, Armonk, NY, USA). One-way analysis of variance was used to detect the differences in changes between the groups of various treatments after establishing that the data were normally distributed as well as equivalency of variances. $\mathrm{P}<0.05$ was considered to indicate a statistically significant difference.

\section{Results}

Content of TSG (\%) and CAQ (\%) in the PM extracts. Based on the analytical method mentioned above, TSG, emodin and physcion were well separated in the HPLC chromatograms (Figs. 2 and 3). The contents of the $30 \%$ ethanolic eluate and the crude extract of PM were analysed from data obtained from triplicate experiments. For quantitative determination of TSG and CAQ in the PM extracts, TSG solutions at concentrations ranging from 0.625 to $20 \mu \mathrm{g} / \mathrm{ml}$ were injected and the regression equation was determined to be $y=68.514 x+35.168$, $\mathrm{r}=0.9998$. The solution for emodin was used at concentrations ranging from 1.25 to $20 \mu \mathrm{g} / \mathrm{ml}$. Physcion was determined by injecting five concentrations ranging from 5 to $80 \mu \mathrm{g} / \mathrm{ml}$. In the $30 \%$ ethanolic eluent of PM, the content of TSG was
$9.20 \pm 1.18 \%$ and that of CAQ was $0.15 \pm 1.08 \%$, and in the crude extract of PM, the content of TSG was $2.23 \pm 1.26 \%$ and that of CAQ was $0.03 \pm 1.32 \%$ (Table I, Figs. 2 and 3 ).

Effects of PM on alterations of body and vital organ weight. Alterations in the body weight of rats were not significantly different between any of the groups during the initial stage of the experiment, while the weight of rats in the prednisone group decreased significantly in the fourth week compared with that in the control group ( $\mathrm{P}<0.05$; Figs. 4 and 5). Conversely, the body weight of rats in the $30 \%$ ethanolic eluate of PM (M) and crude extract of PM $(\mathrm{H})$ groups was increased compared with that in the prednisone group $(\mathrm{P}<0.05)$. Of note, the weight of adrenal gland, liver, tibialis anterior muscle, ovaries and uterus in the prednisone group was also decreased compared with that in the control group $(\mathrm{P}<0.05)$. Compared with that in the prednisone group, the weight of the thymus of male rats and the weight of the ovaries in female rats was increased in the CAL group $(\mathrm{P}<0.05)$. The weight of body, thymus and tibialis anterior muscle in male and female rats as well as that of the ovaries increased in the $30 \%$ ethanolic eluate of PM (M) and crude extract of PM $(\mathrm{H})$ groups compared with that in the prednisone group $(\mathrm{P}<0.05$; Tables II and III).

Detection of $2 \mathrm{D}$ images. According to $2 \mathrm{D}$ reconstruction images of sagittal sections of the right femur and the $\mathrm{LV}_{4}$ of rats in the prednisone group, the microarchitecture of bone trabecula showed an irregular and smaller shape compared with that in the control group. Contrarily, the microarchitecture of the bone trabecula of rats in the CAL group, the $30 \%$ ethanolic eluate of PM (M, L) and the crude extract of PM $(\mathrm{H}, \mathrm{M})$ groups exhibited a more regular shape than those in the prednisone group. Based on evidence from coronal section images, the density of the bone trabeculae had decreased in the prednisone group, which was partly recovered in the CAL, $30 \%$ ethanolic eluent of PM (M, L) and crude extract of PM $(H$, $\mathrm{M})$ groups. As shown in the cross section images, the thickness of the conical bone in these groups was also higher than that in the prednisone group (Figs. 6-9 and Tables IV-VII).

Analysis of $3 D$ reconstruction images. After the microarchitecture of the bone trabecula was observed in the 2D reconstructed images, $3 \mathrm{D}$ reconstructed images of the right femur and the $\mathrm{LV}_{4}$ were generated for each group of rats (Figs. 10-13). The segmentation (SEG) images showed a regular shape, uniform thickness and dense arrangement of bone trabecula in the control group. By comparison, in the prednisone group, irregular shapes, reduced thickness and only a scattered abundance of bone trabecula was identified. In the CAL group, $30 \%$ ethanolic eluate of PM (M, L) and crude extract of PM $(\mathrm{H}, \mathrm{M})$ groups, a reduced destruction of bone microarchitecture was shown compared to that in the prednisone group (Figs. 10-13). Separation (SP) images revealed that the red, orange and yellow areas were larger, particularly the red area in the prednisone group compared with that in the control group. It was indicated that SP was increased and the distance of bone trabecula had become larger. In the CAL, 30\% ethanolic eluate of PM (M, L) and crude extract of PM $(\mathrm{H})$ groups, the green area was larger than that in the prednisone group, wile the red, orange and yellow areas 
Table I. Content of TSG and CAQ in the extracts prepared (\%).

\begin{tabular}{lcccc}
\hline Group & TSG & FAQ & TAQ & CAQ=TAQ-FAQ \\
\hline $30 \%$ ethanol eluate of PM & $9.20 \pm 1.08$ & $0.19 \pm 1.08$ & $0.34 \pm 1.08$ & $0.15 \pm 1.08$ \\
Crude PM extract & $2.23 \pm 1.26$ & $0.11 \pm 1.32$ & $0.14 \pm 1.32$ & $0.03 \pm 1.32$ \\
\hline
\end{tabular}

PM, Polygonum multiflorum; TSG, 2, 3, 5, 4'-tetrahydroxystilbene-2-O- $\beta$-glucoside; CAQ, combined anthraquinone; TAQ, total anthraquinone; FAQ, free anthraquinone.

were smaller. It was indicated that the distance between bone trabecula had become shorter in these groups compared with that in the prednisone group. In the thickness (TH) images, the green area in the prednisone group had become more lager compared with that in the control group, while the red, orange and yellow areas had decreased in female rats compared those in the control group, indicating that the thickness of bone trabecula had decreased (Fig. 11). It was found that the area of red, orange and yellow in the CAL, 30\% ethanolic eluate of PM (M, L) and crude extract of PM (H, M, L) groups was larger compared with that in the prednisone group, indicating that the thickness of bone trabecula was increased; however, this phenomenon was not obvious in the femurs of male rats (Fig. 10).

Effects of PM on bone microarchitecture. The present study assessed the microarchitecture of bone trabecula in the sagittal section images, the density of bone trabeculae in the coronal section images and the thickness of conical bone in the cross sectional images. At the same time, the present study observed the thickness of bone trabecula in TH images, separation of bone trabecula in SP images and BV/TV of bone trabecula in SEG images. According to measurements of the right femur and the $\mathrm{LV}_{4}$ by $3 \mathrm{D}$ reconstruction, the parameters of bone microarchitecture were calculated for the analysis of the alterations of bone mass and structural characteristics. As shown in Tables IV-VII, BV/TV and density in the prednisone group were obviously damaged compared with those in the control group $(\mathrm{P}<0.05)$, while CAL significantly counteracted the deteriorating influence of prednisone on the bone tissue microarchitecture. $30 \%$ ethanolic eluate of PM (M, L) and crude extract of the (H) also significantly improved the bone microstructure by increasing the BV/TV of the femur compared with that in the prednisone group $(\mathrm{P}<0.05)$, but not to the same extent as CAL. 30\% ethanolic eluate of PM $(\mathrm{M}, \mathrm{L})$ and crude extract of PM $(\mathrm{H}, \mathrm{M}, \mathrm{L})$ were shown to improve the bone microstructure by increasing the $\mathrm{BV} / \mathrm{TV}$ of $\mathrm{LV}_{4}$ compared with that in the prednisone group $(\mathrm{P}<0.05)$.

Effects of PM on BMD. Compared with the control group, the BMD of the distal femur and $\mathrm{LV}_{4}$ in male rats of the prednisone group was significantly decreased $(\mathrm{P}<0.05)$, while this was not found in female rats. Compared with the prednisone group, CAL significantly improved the BMD of the distal femur of all rats and the BMD of $\mathrm{LV}_{4}$ of male rats. Although the other administration groups showed no significant differences in BMD compared to that in the prednisone group, a

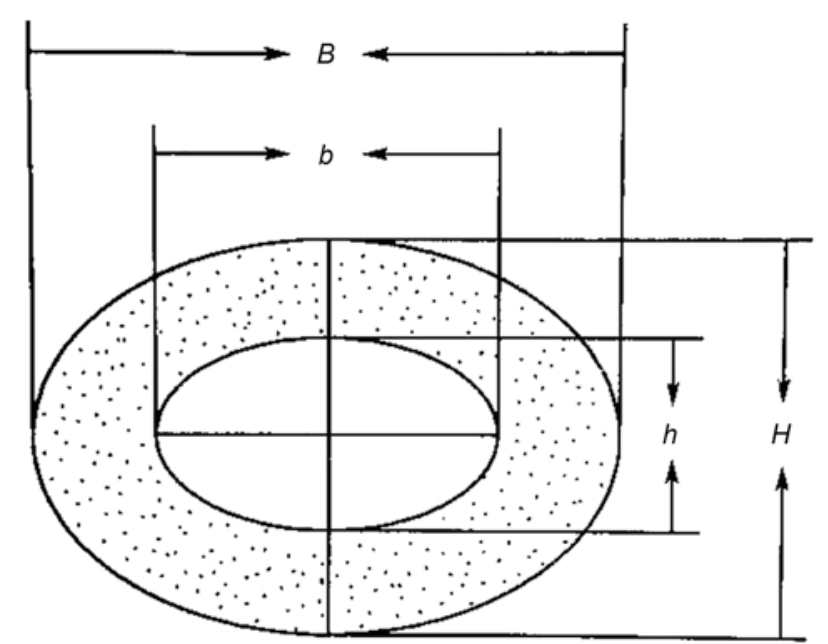

Figure 1. Cross-sectional diagram of the femur.

trend towards increased BMD was observed in female and male groups (Tables VIII and IX).

Effects of PM on biomechanical properties. In the prednisone group, biomechanical properties of femur and $\mathrm{LV}_{5}$ were found to be deteriorated, as evidenced by a decreased maximum load compared with that in the control group of male and female rats. CAL significantly improved the biomechanical properties of elastic load of femur and stiffness compared with those in the prednisone group $(\mathrm{P}<0.05)$. The biomechanical properties of elastic load and stiffness of femur and $L_{5}$ were significantly increased by the crude extract of PM $(\mathrm{H} ; \mathrm{P}<0.05)$. The effects of $30 \%$ ethanolic eluate of PM (M, L) and crude extract of PM $(\mathrm{H})$ were not statistically significant, while there was a trend toward increased biomechanical function of femur and $\mathrm{LV}_{5}$ in female and male rats (Tables X and XI).

\section{Discussion}

An increasing number of clinical studies have evidenced the deleterious effect of GC on skeletal tissue, contributing to the development and progession of osteoporosis. The present study demonstrated that GC exerts a series of deleterious actions on bone tissue in male as well as female rats. A previous study by our group has demonstrated that extracts of PM exert a preventive effect on the bones of rats with ovariectomy-induced osteoporosis (19). The present study demonstrated that extracts of PM have a protective effect on BMD, bone mass, bone 

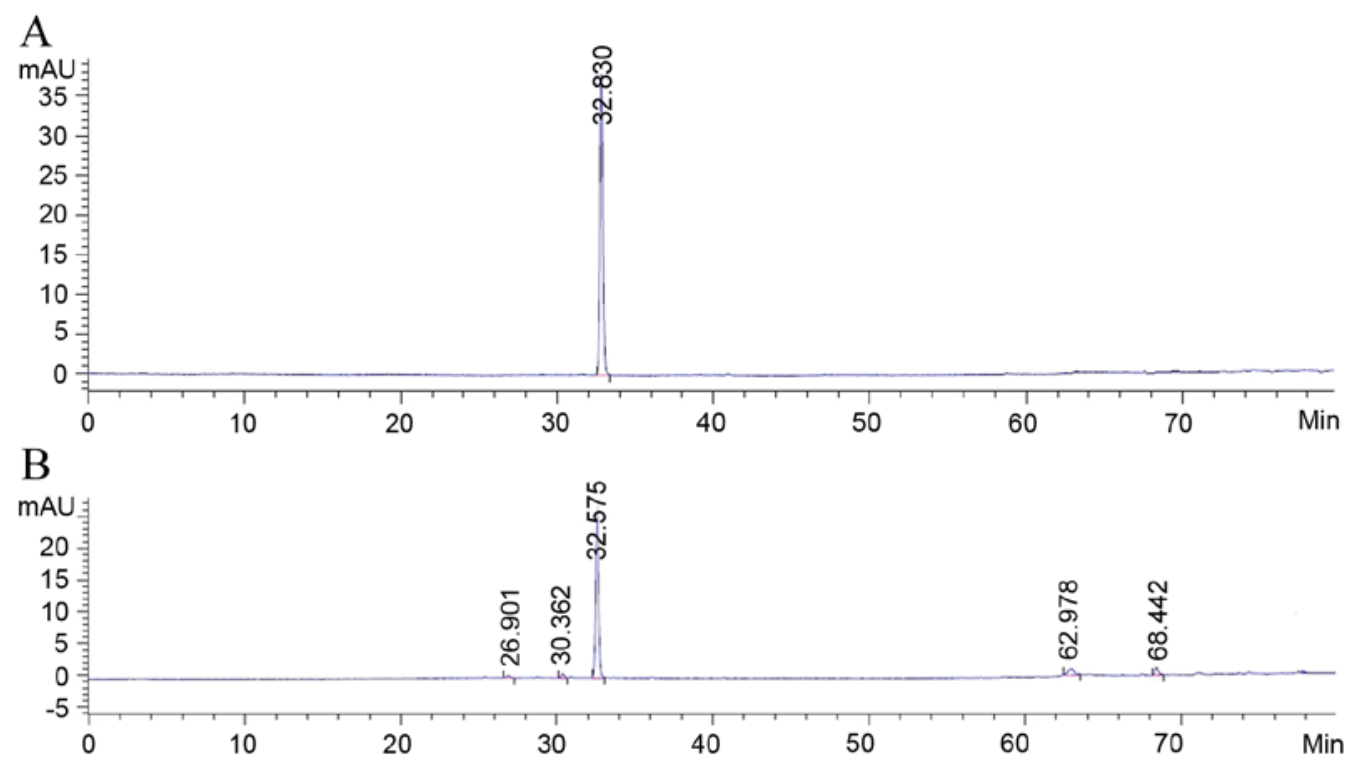

Figure 2. High-performance liquid chromatograms of standards and samples of Polygonum multiflorum. (A) Standard solution of 2,3,5,4'-tetrahydroxystilben e-2-O- $\beta$-glucoside; (B) sample solution of Polygonum multiflorum.
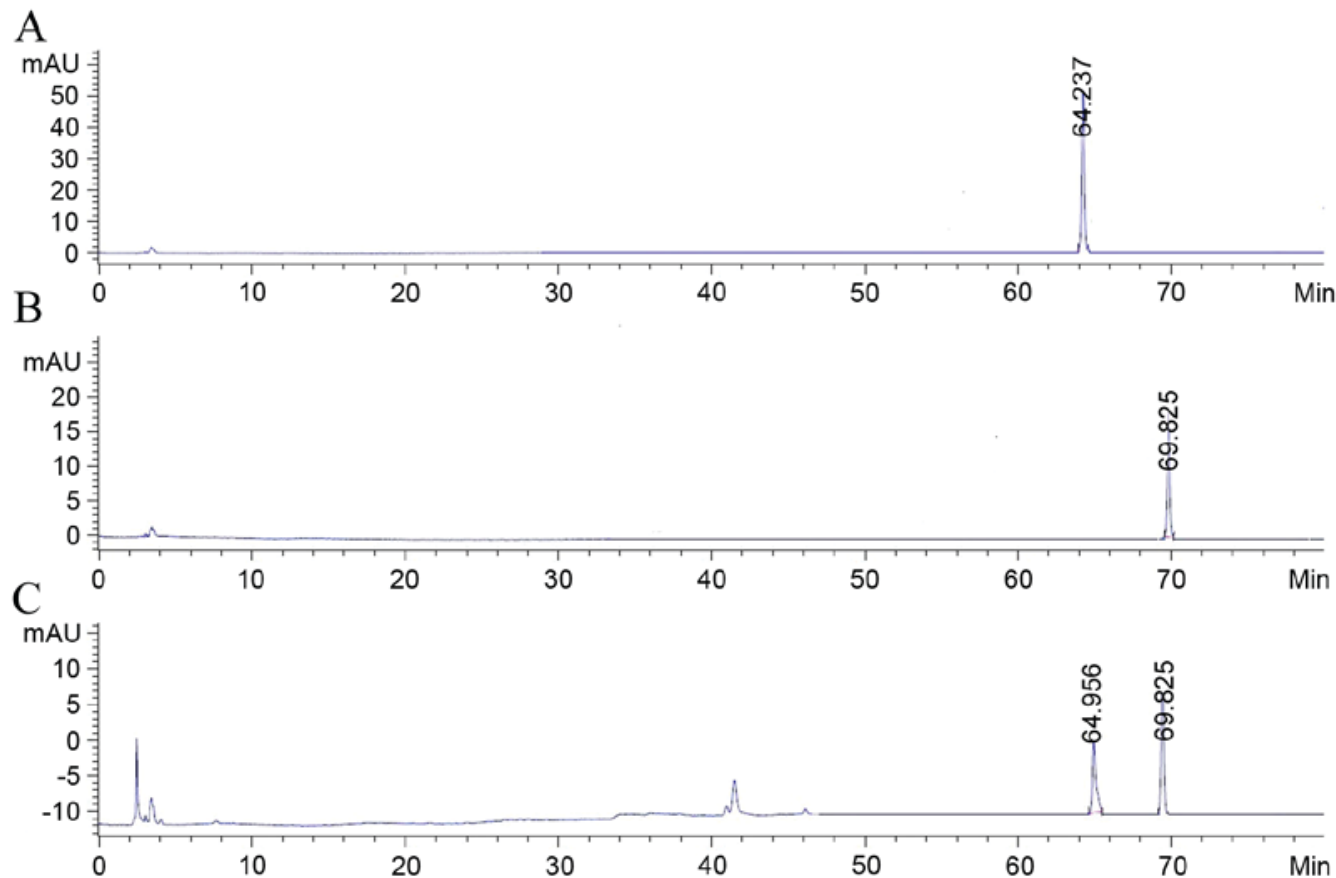

Figure 3. High-performance liquid chromatograms of standards and samples of Polygonum multiflorum. (A) Standard solution of emodin; (B) standard solution of physcion; (C) sample solution. Peaks: 1, emodin; 2, physcion.

microarchitecture and bone strength in rats exposed to prednisone, indicating that PM has therapeutic potential in GIO.

Glucocorticoids are effective anti-inflammatory and immunomodulatory agents, which secondarily contribute to dysfunction of bone metabolism, increases of bone fragility and the ultimate consequence of bone fractures (2). According to the results of the present study the BV/TV, a significant skeletal parameter, was decreased in the distal femur and $\mathrm{LV}_{4}$, suggesting an obvious reduction in the content of the relative volume fraction of cancellous bone in bone tissue of rats treated with prednisone. Accordingly, the alterations in the trabecular architecture of the skeleton also consolidated the deleterious action of GC in terms of the direct visual method of 3D SEG imaging (34). The present study found that abnormal bone microstructure as well as a decrease in BMD and biomechanical properties were accompanied with weight loss of body, skeletal muscle and immune organs in rats treated with prednisone. The possible mechanisms of GIO are increased apoptosis of osteoblasts and mature osteocytes via activation of caspase 3 (35-37). In addition, $\mathrm{GC}$-induced repression of bone morphogenetic protein-2 and expression of core binding factor a1, an osteoblast-specific gene, which is a critical factor for osteoblastogenesis and osteocalcin, may lead to reduced osteoblast 


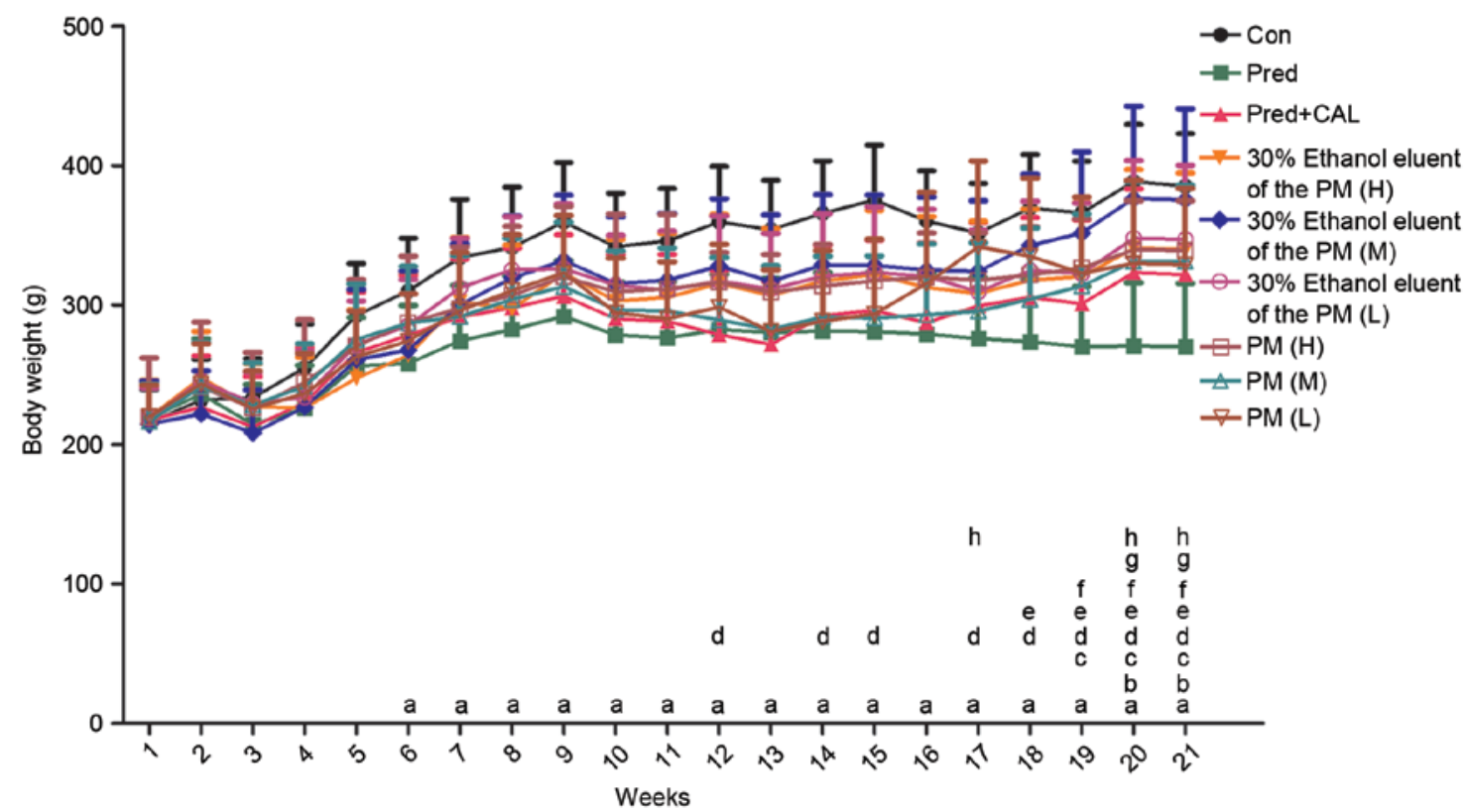

Figure 4. Changes in the weight in male rats in different groups over an administration period of 21 weeks. ${ }^{a} \mathrm{P}<0.05$ vs. CON; ${ }^{b} \mathrm{P}<0.05 \mathrm{CAL}$ vs. Pred; ${ }^{\mathrm{P}} \mathrm{P}<0.05$ $30 \%$ ethanolic eluate of PM (H) vs. Pred; ${ }^{d} \mathrm{P}<0.0530 \%$ ethanolic eluate of $\mathrm{PM}(\mathrm{M})$ vs. Pred; ${ }^{\mathrm{e}} \mathrm{P}<0.0530 \%$ ethanolic eluate of $\mathrm{PM}$ (L) vs. Pred; ${ }^{\mathrm{f}} \mathrm{P}<0.05$ crude extract of PM (H) vs. Pred; ${ }^{\mathrm{P}} \mathrm{P}<0.05$ crude extract of $\mathrm{PM}(\mathrm{M})$ vs. Pred; ${ }^{\mathrm{h}} \mathrm{P}<0.05$ crude extract of $\mathrm{PM}$ (L) vs. Pred. Pred, prednisone; PM, Polygonum multiflorum; $\mathrm{H}$, high dose; $\mathrm{M}$, medium dose; L, low dose; CAL, calcitriol; CON, control.

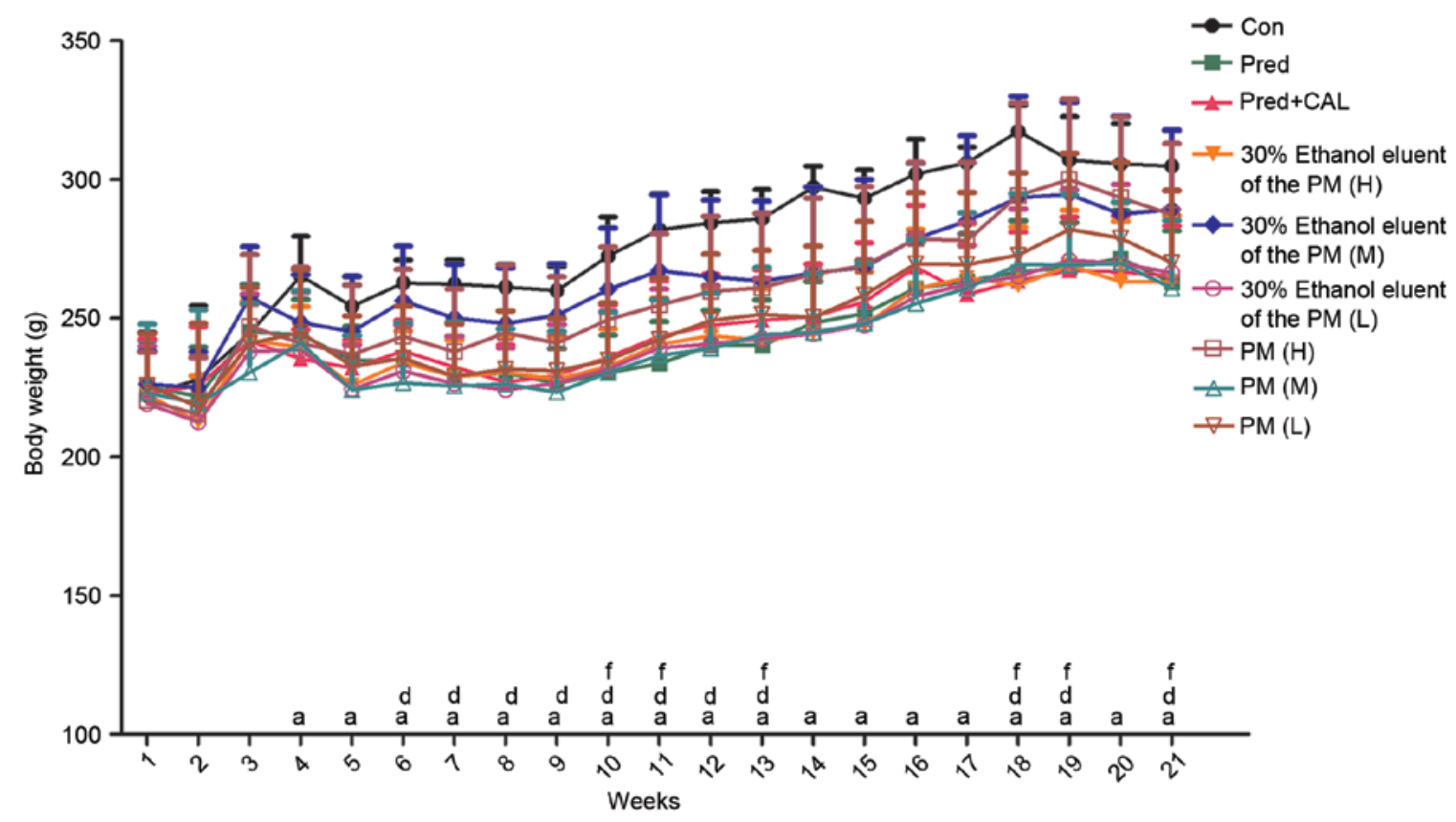

Figure 5. Changes in the weight of the female rats in different groups over the administration period ( 21 weeks). ${ }^{a} \mathrm{P}<0.05$ vs. CON; ${ }^{\mathrm{P}} \mathrm{P}<0.05 \mathrm{CAL}$ vs. Pred; ${ }^{c} \mathrm{P}<0.0530 \%$ ethanolic eluate of $\mathrm{PM}(\mathrm{H})$ vs. Pred; ${ }^{\mathrm{d}} \mathrm{P}<0.0530 \%$ ethanolic eluate of $\mathrm{PM}(\mathrm{M})$ vs. Pred; ${ }^{\mathrm{e}} \mathrm{P}<0.0530 \%$ ethanolic eluate of $\mathrm{PM}(\mathrm{L})$ vs. Pred; ${ }^{\mathrm{f}} \mathrm{P}<0.05$ crude extract of PM (H) vs. Pred; ${ }^{\mathrm{P}} \mathrm{P}<0.05$ crude extract of PM (M) vs. Pred; ${ }^{\mathrm{h}} \mathrm{P}<0.05$ crude extract of PM (L) vs. Pred. Pred, prednisone; PM, Polygonum multiflorum; $\mathrm{H}$, high dose; $\mathrm{M}$, medium dose; $\mathrm{L}$, low dose; $\mathrm{CAL}$, calcitriol; $\mathrm{CON}$, control.

proliferation and differentiation $(35,38)$. Studies have shown that GCs stimulate bone resorption, which may be associated with the stimulation of osteoclast differentiation and their capacity to bind to the bone surface by altering the expression of $N$-acetylglucosamine and $N$-acetylgalactosamine (35).

PM is of high medical value in Traditional Chinese Medicine (39). It has been reported that a PM decoction and aqueous extracts of PM have preventive effects on bone loss in ovariectomized rats by inhibiting bone resorption (19). However, the preventive effect of PM on bone loss in rats with prednisone-induced osteoporosis has remained to be elucidated. To the best of our knowledge, the present study was the first to show that extracts of PM, whose major active components are TSG and CAQ, are able to reduce bone loss 

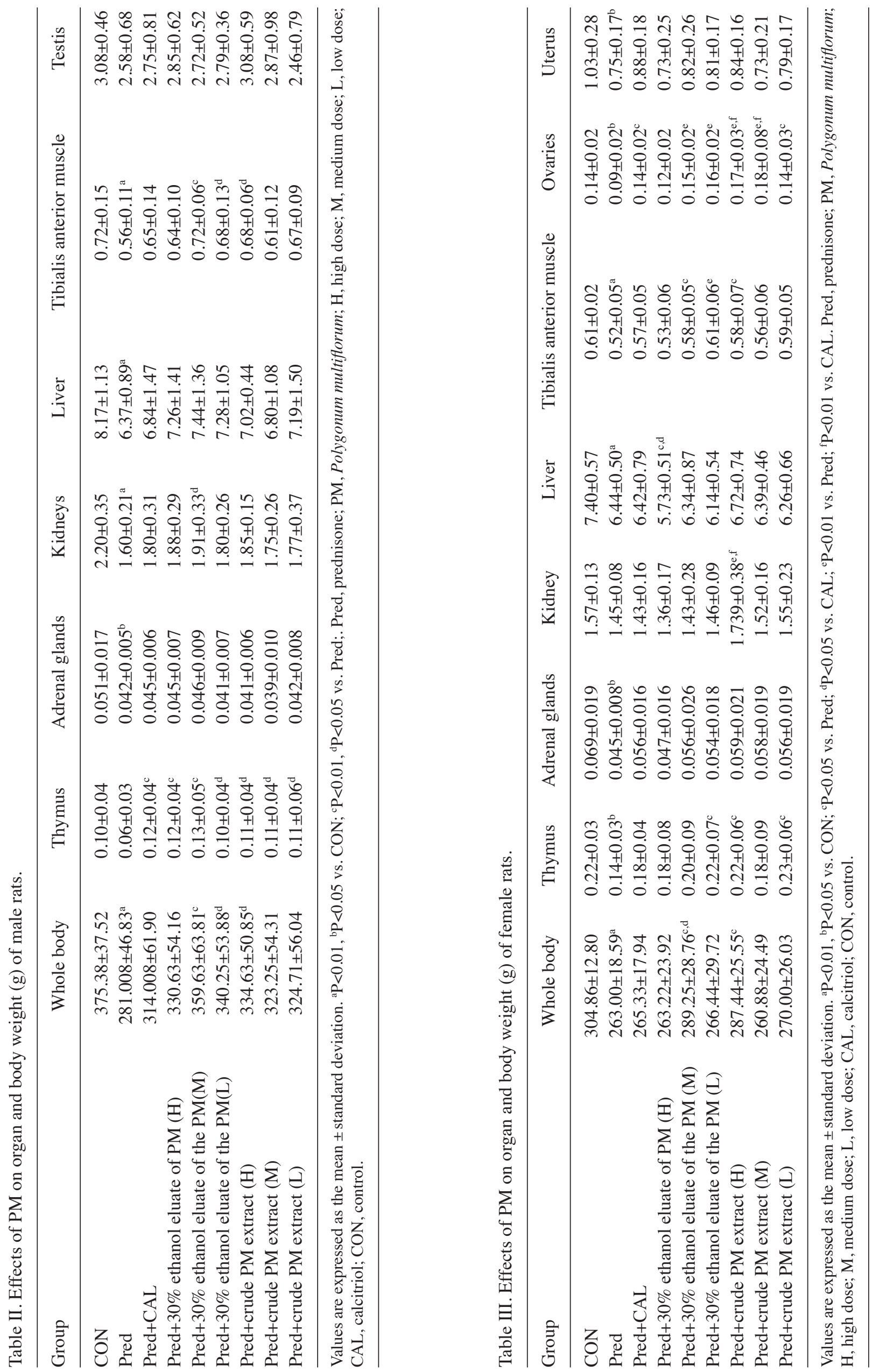
A Sagittal section
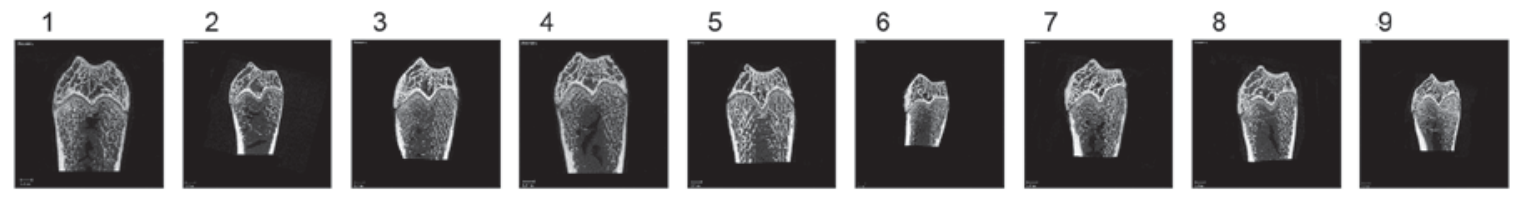

B Coronal section
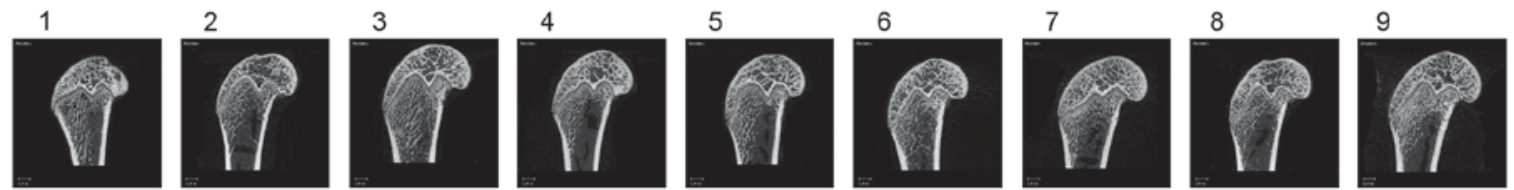

C Cross section
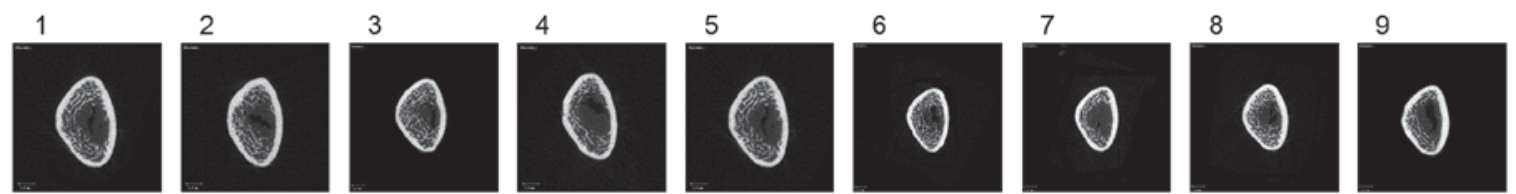

Figure 6. Two-dimensional images of the right femurs of male rats. (A) Saggital, (B) coronal and (C) cross sections. Groups: 1, control; 2 , Pred (6 mg/kg/day); 3 , Pred + calcitriol $(0.045 \mu \mathrm{g} / \mathrm{kg} /$ day) $; 4$, Pred $+30 \%$ ethanolic eluate of PM (high dose; $400 \mathrm{mg} / \mathrm{kg} / \mathrm{day}) ; 5$, Pred $+30 \%$ ethanolic eluate of PM (medium dose; $200 \mathrm{mg} / \mathrm{kg} / \mathrm{day}) ; 6$, Pred $+30 \%$ ethanolic eluate of PM (low dose; $100 \mathrm{mg} / \mathrm{kg} /$ day); 7 , Pred + crude extract of PM (H; $400 \mathrm{mg} / \mathrm{kg} / \mathrm{d}) ; 8$, Pred + crude extract of PM (M; 200 mg/kg/day); 9, Pred + crude extract of PM (L; 100 mg/kg/day). Magnification, x12. Pred, prednisone; PM, Polygonum multiflorum.

A Sagittal section
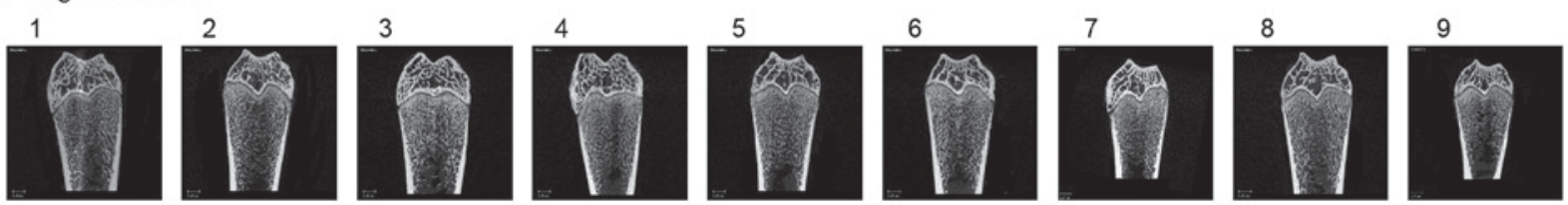

B Coronal section
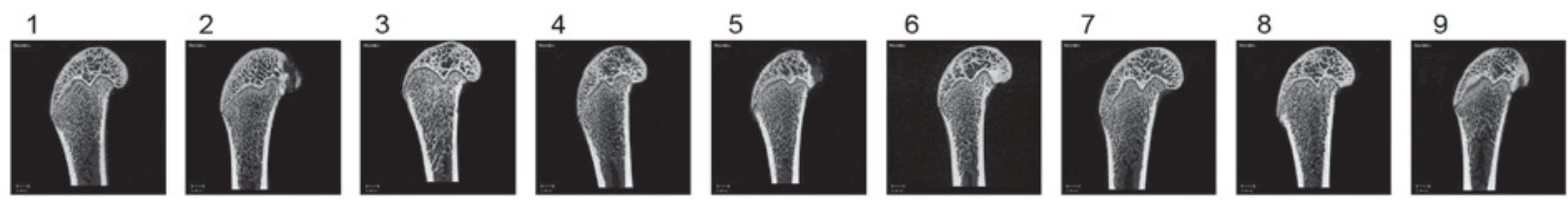

C Cross section
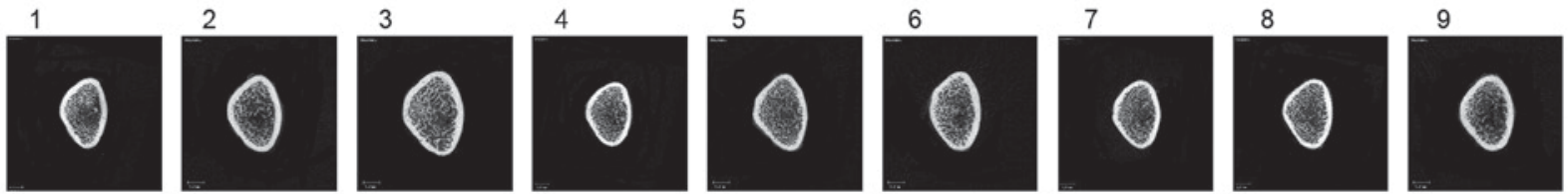

Figure 7. Two-dimensional images of the right femurs of female rats. (A) Saggital, (B) coronal and (C) cross sections. Groups: 1, control; 2 , Pred (6 mg/kg/day); 3, Pred + calcitriol $(0.045 \mu \mathrm{g} / \mathrm{kg} / \mathrm{day}) ; 4$, Pred $+30 \%$ ethanolic eluate of PM (high dose; $400 \mathrm{mg} / \mathrm{kg} / \mathrm{day}$ ); 5 , Pred $+30 \%$ ethanolic eluate of PM (medium dose; $200 \mathrm{mg} / \mathrm{kg} / \mathrm{day}) ; 6$, Pred + 30\% ethanolic eluate of PM (low dose; $100 \mathrm{mg} / \mathrm{kg} / \mathrm{day}) ; 7$, Pred + crude extract of PM (H; $400 \mathrm{mg} / \mathrm{kg} / \mathrm{d}) ; 8$, Pred + crude extract of PM (M; $200 \mathrm{mg} / \mathrm{kg} / \mathrm{day}) ; 9$, Pred + crude extract of PM (L; $100 \mathrm{mg} / \mathrm{kg} /$ day). Magnification, x12. Pred, prednisone; PM, Polygonum multiflorum.

in prednisone-induced rats. It was shown that the content of TSG and CAQ in the $30 \%$ ethanolic eluate of PM was higher than that in the crude extract of the PM. It has been previously reported that herbal samples a higher content of TSG and CAQ contain greater amounts of the pharmacologically active components of PM and are of better quality regarding clinical application (25). In the present study, HPLC was a convenient and effective approach to assess the quality of PM. The results showed that this method has a good reproducibility with a relative standard deviation of $\leq 2.0 \%$.
The present study investigated the preventive effects of PM on prednisone-induced bone loss through assessing BMD, biomechanical properties and microstructure. In the $30 \%$ ethanolic eluate of PM (M, L) and crude extract of PM (H) groups, bone microarchitecture was improved, and BMD, biomechanical properties and the weight of thymus and ovaries were increased compared to those in the prednisone group, therefore producing an inhibitory effect on GIO, which was, however, not as high as that of CAL. It was also shown that the differences between the $30 \%$ ethanolic eluate of PM $(\mathrm{H})$ group and the prednisone 
A Sagittal section
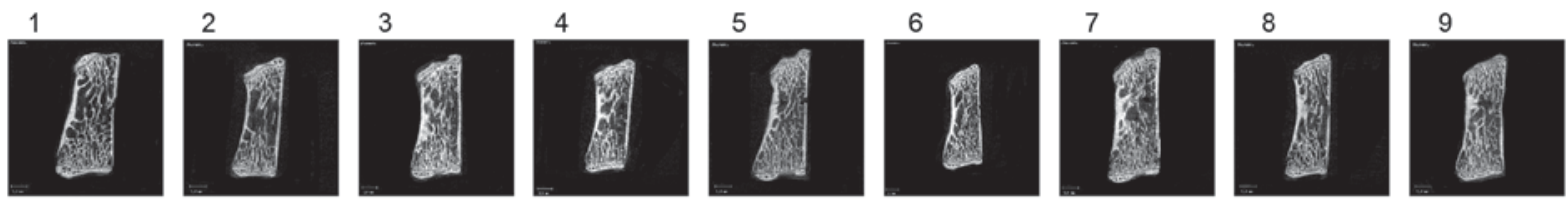

B Coronal section
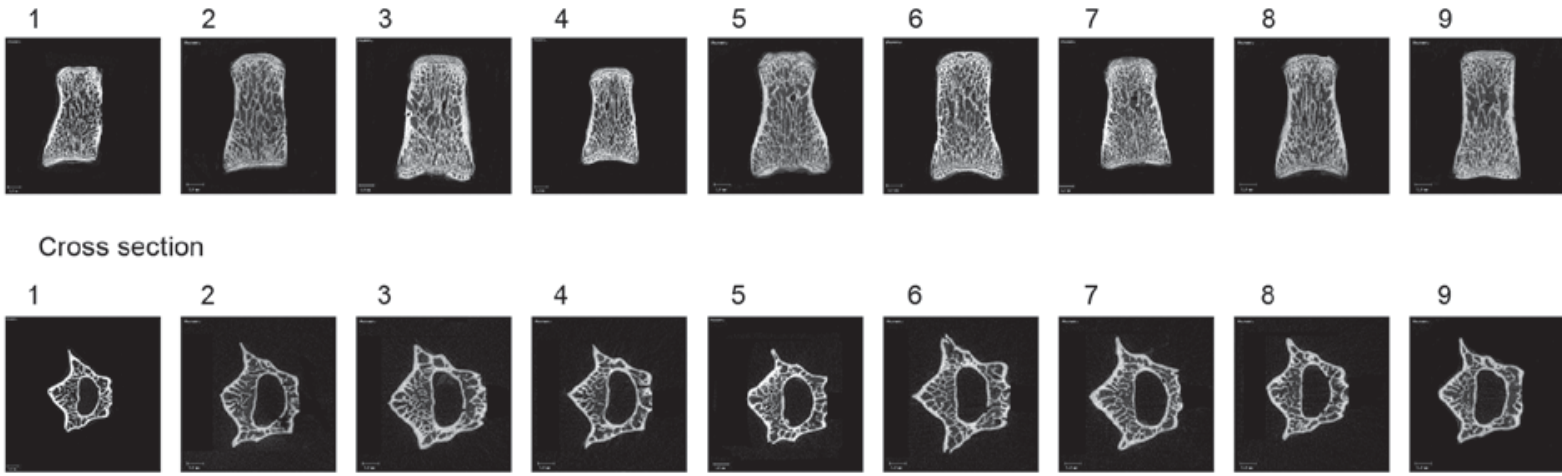

C Cross section
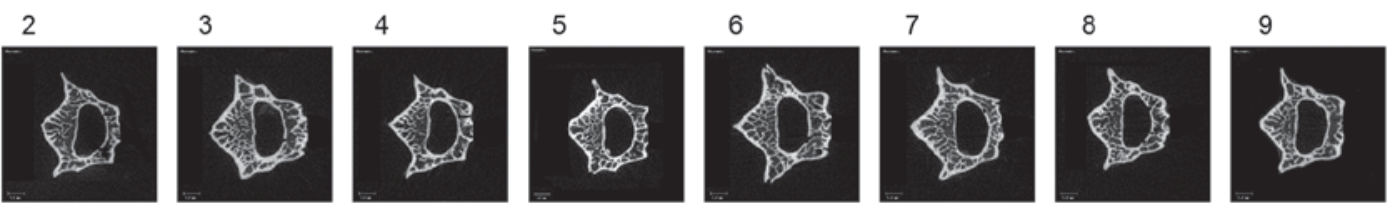

Figure 8. Two-dimensional images of the $\mathrm{LV}_{4}$ in male rats. (A) Saggital, (B) coronal and (C) cross sections. Groups: 1, control; 2, Pred (6 mg/kg/day); 3 , Pred + calcitriol ( $0.045 \mu \mathrm{g} / \mathrm{kg} / \mathrm{day}) ; 4$, Pred $+30 \%$ ethanolic eluate of PM (high dose; $400 \mathrm{mg} / \mathrm{kg} /$ day); 5 , Pred + 30\% ethanolic eluate of PM (medium dose; $200 \mathrm{mg} / \mathrm{kg} /$ day); 6, Pred + 30\% ethanolic eluate of PM (low dose; $100 \mathrm{mg} / \mathrm{kg} /$ day); 7, Pred + crude extract of PM (H; $400 \mathrm{mg} / \mathrm{kg} / \mathrm{d}$ ); 8, Pred + crude extract of PM (M; 200 mg/kg/day); 9, Pred + crude extract of PM (L; 100 mg/kg/day). Magnification, x12. Pred, prednisone; PM, Polygonum multiflorum.

A Sagittal section
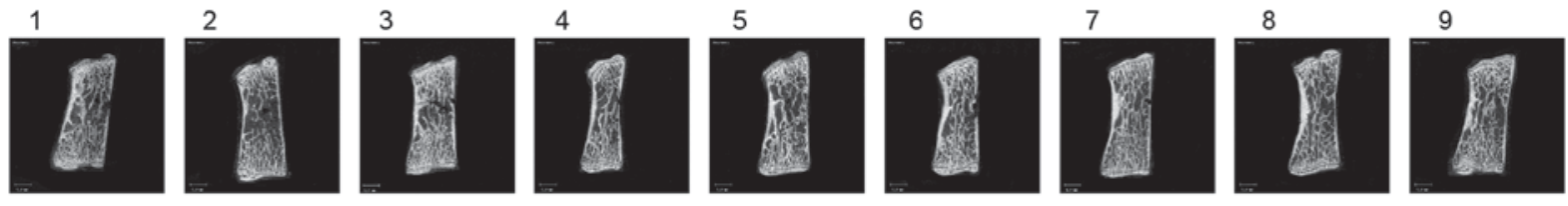

B Coronal section
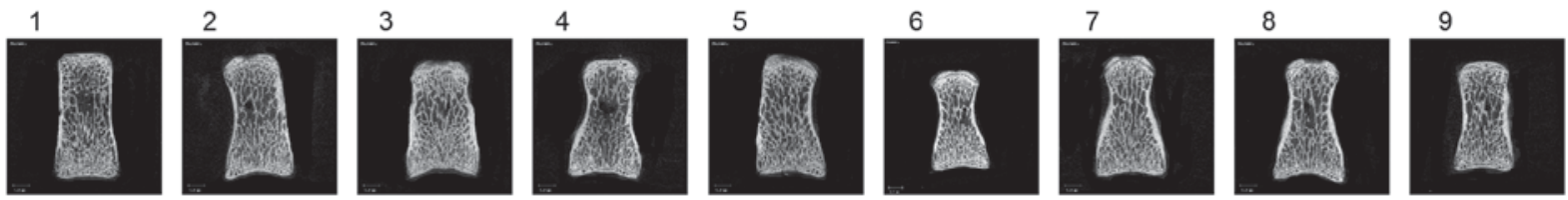

C Cross section
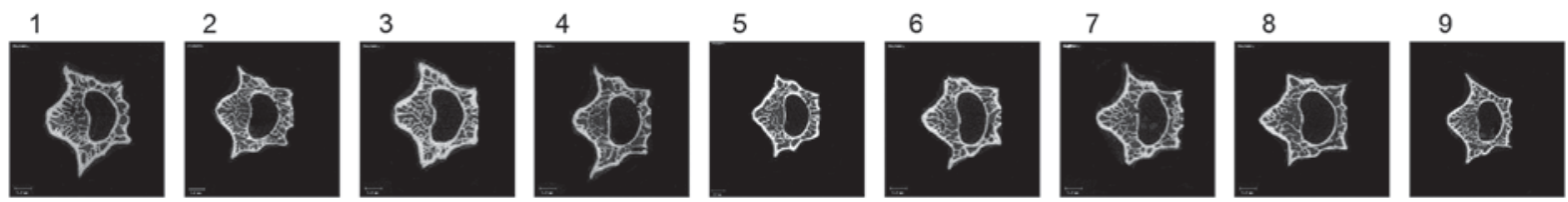

Figure 9. Two-dimensional images of the $\mathrm{LV}_{4}$ in female rats. (A) Saggital, (B) coronal and (C) cross sections. Groups: 1, control; 2, Pred (6 mg/kg/day); 3 , Pred + calcitriol $(0.045 \mu \mathrm{g} / \mathrm{kg} / \mathrm{day}) ; 4$, Pred $+30 \%$ ethanolic eluate of PM (high dose; $400 \mathrm{mg} / \mathrm{kg} /$ day); 5 , Pred + 30\% ethanolic eluate of PM (medium dose; $200 \mathrm{mg} / \mathrm{kg} /$ day); 6, Pred + 30\% ethanolic eluate of PM (low dose; $100 \mathrm{mg} / \mathrm{kg} /$ day); 7 , Pred + crude extract of PM (H; $400 \mathrm{mg} / \mathrm{kg} / \mathrm{d}) ; 8$, Pred + crude extract of PM (M; 200 mg/kg/day); 9, Pred + crude extract of PM (L; 100 mg/kg/day). Pred, prednisone; PM, Polygonum multiflorum.

group were not statistically significant. According to previous studies, the anti-osteoporosis effects of PM may be associated with the following mechanisms (19,40,41): i) Improved activity of $1 \alpha$-hydroxylase, increased content of calcium, phosphorus and hydroxyproline in bone tissue and reduced bone loss by activating alkaline phosphatase. ii) Increased content of malondialdehyde by activation of superoxide dismutase and anti-oxidant enzymes to prevent aging. iii) Improved kidney morphology with reduction of glomerular and tubular fibrosis. iv) TSG is a plant-derived estrogen with bioactivity, which may be used for estrogen replacement therapy. Therefore, PM may be considered as an ideal candidate for the prevention and treatment of GIO due to being economical, convenient and having a high clinical value.

Of note, the present study found that female rats may be more sensitive to prednisone treatment by gavage for 21 weeks than male rats to establish a model of osteoporosis. This was mainly evidenced by the destruction of the bone microarchitecture (BV/TV), which decreased by 28.6 and $14.0 \%$ in the femur and $\mathrm{LV}_{4}$, respectively, in male rats, while decreasing by 

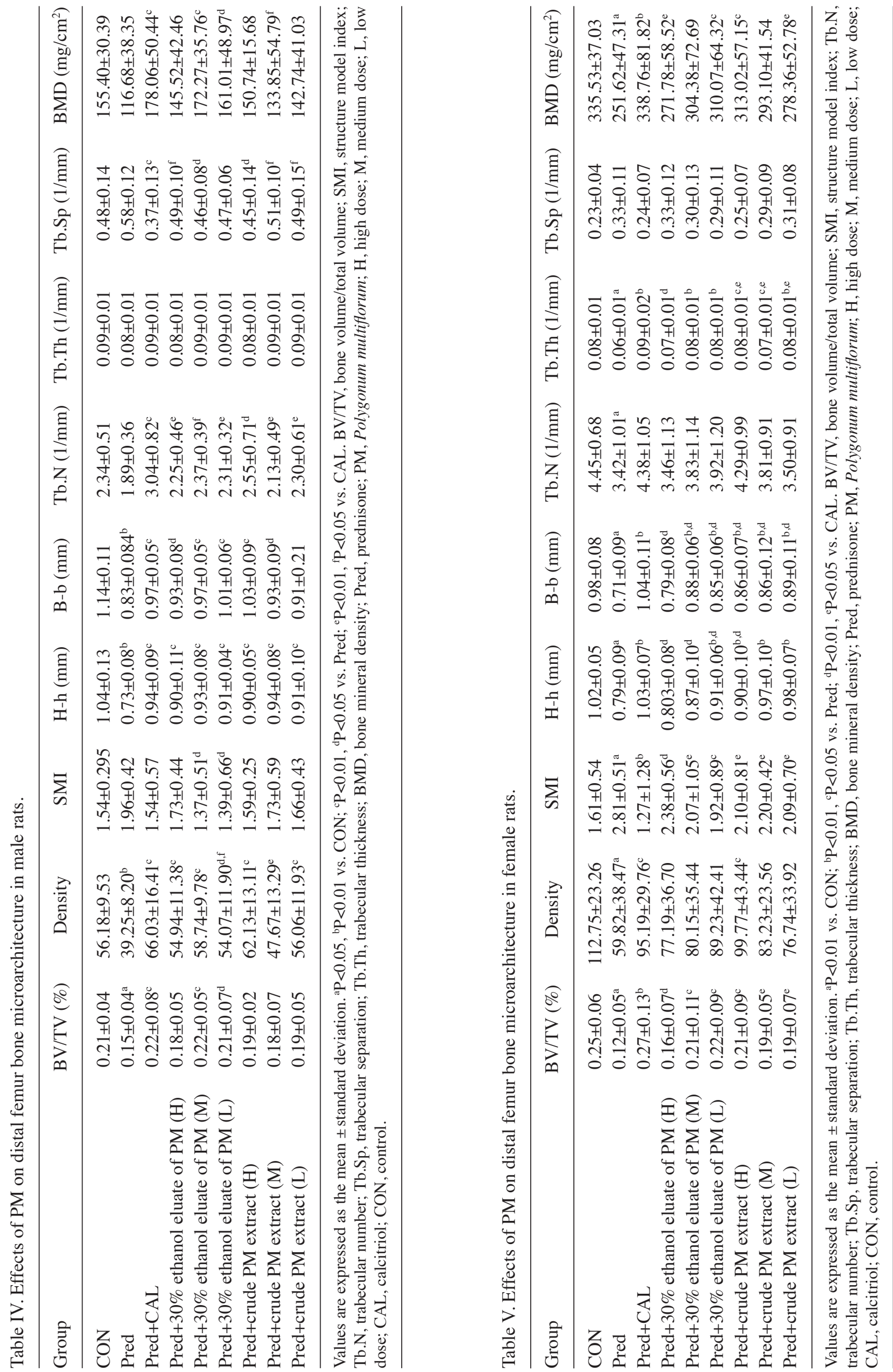

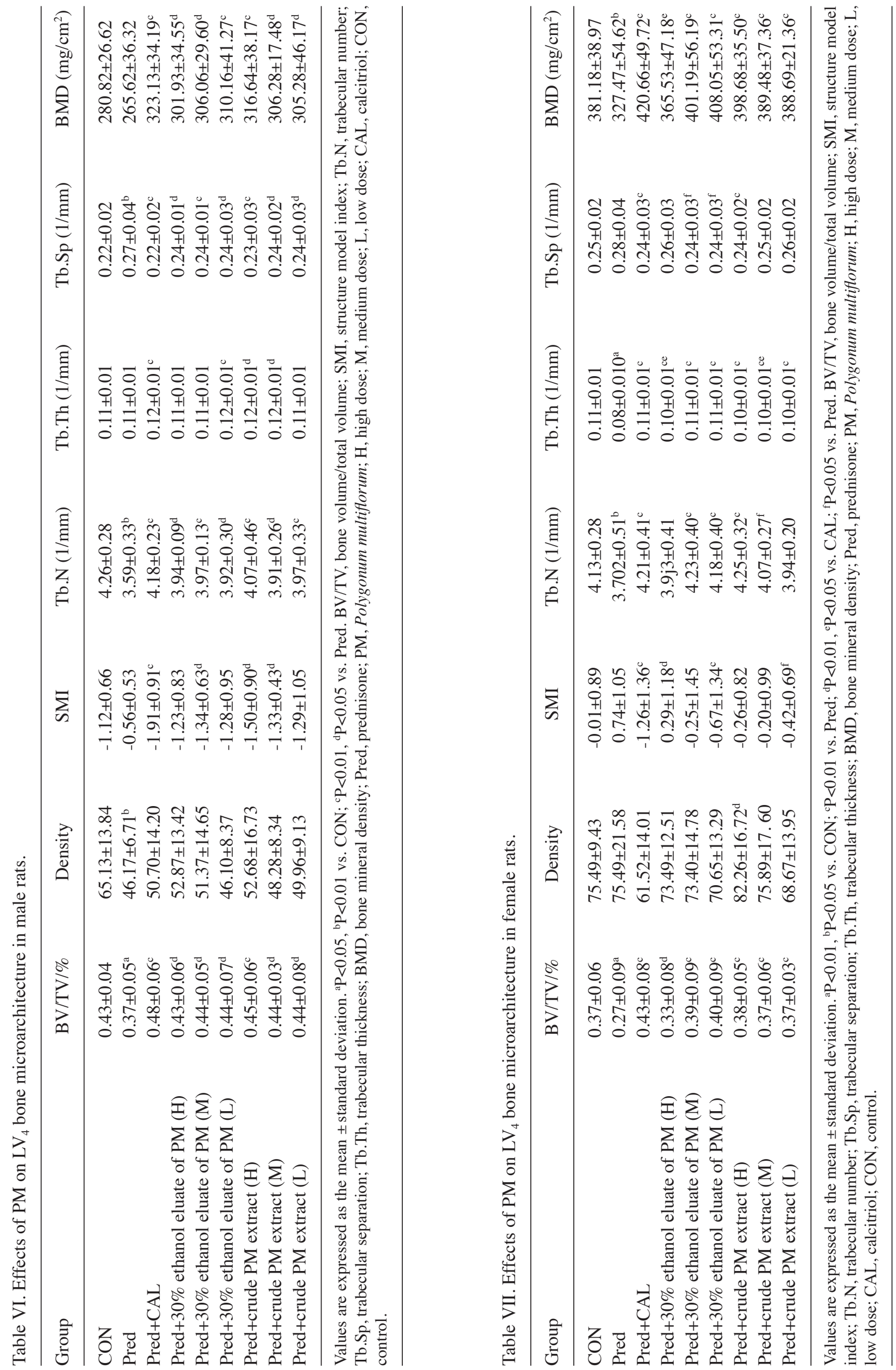
A SEG

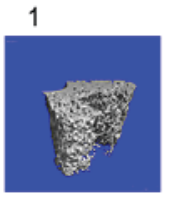

2
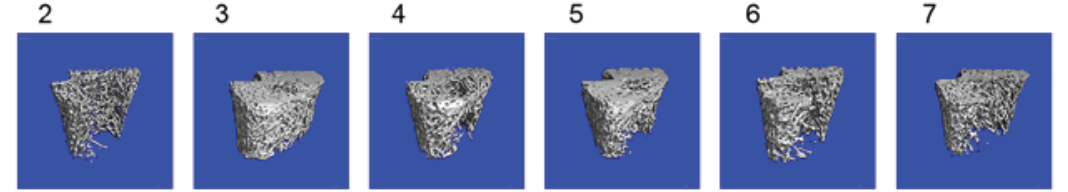

8

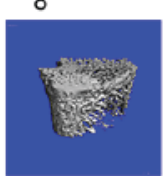

9

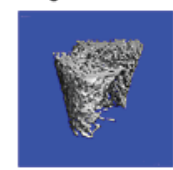

B SP
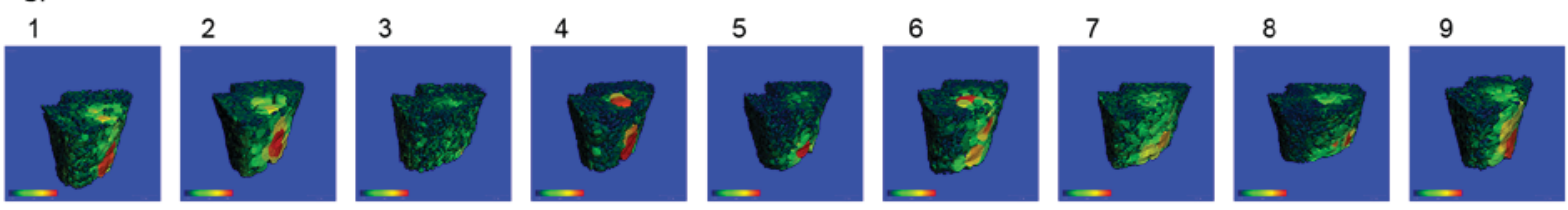

C $\mathrm{TH}$
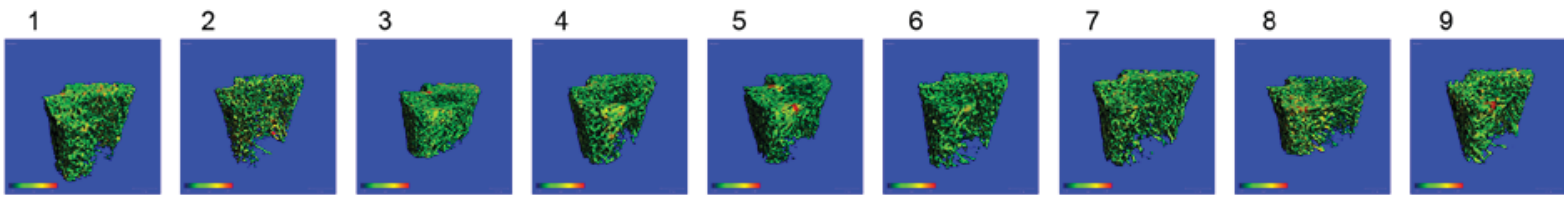

Figure 10. Three-dimensional reconstructed images of the right femur in male rats. (A) SEG image, (B) trabecular SP and (C) trabecular TH. Groups: 1 control; 2, Pred (6 mg/kg/day); 3, Pred + calcitriol (0.045 $\mu \mathrm{g} / \mathrm{kg} / \mathrm{day}) ; 4$, Pred $+30 \%$ ethanolic eluate of PM (high dose; $400 \mathrm{mg} / \mathrm{kg} / \mathrm{day}) ; 5$, Pred $+30 \%$ ethanolic eluate of PM (medium dose; $200 \mathrm{mg} / \mathrm{kg} /$ day); 6, Pred $+30 \%$ ethanolic eluate of PM (low dose; $100 \mathrm{mg} / \mathrm{kg} / \mathrm{day}$ ); 7, Pred + crude extract of PM (H; $400 \mathrm{mg} / \mathrm{kg} / \mathrm{d}) ; 8$, Pred + crude extract of PM (M; $200 \mathrm{mg} / \mathrm{kg} / \mathrm{day}) ; 9$, Pred + crude extract of PM (L; $100 \mathrm{mg} / \mathrm{kg} / \mathrm{day})$. For SP images, green indicates areas of high bone density and red indicates areas of low bone density. For TH images, green indicates areas of low bone density and red indicates areas of high bone density. Magnification, x30. SEG, segmentation; SP, separation; TH, thickness; Pred, prednisone; PM, Polygonum multiflorum.

A SEG
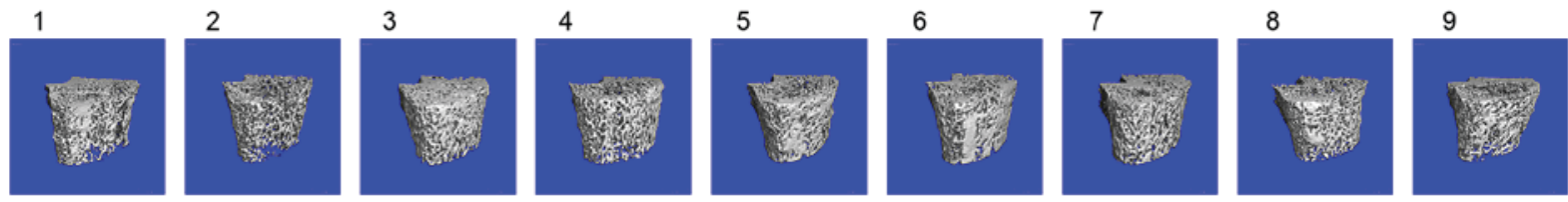

B SP
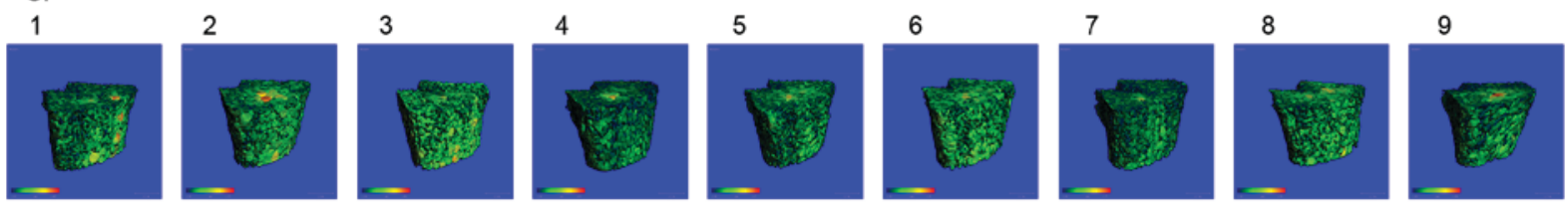

C $\mathrm{TH}$
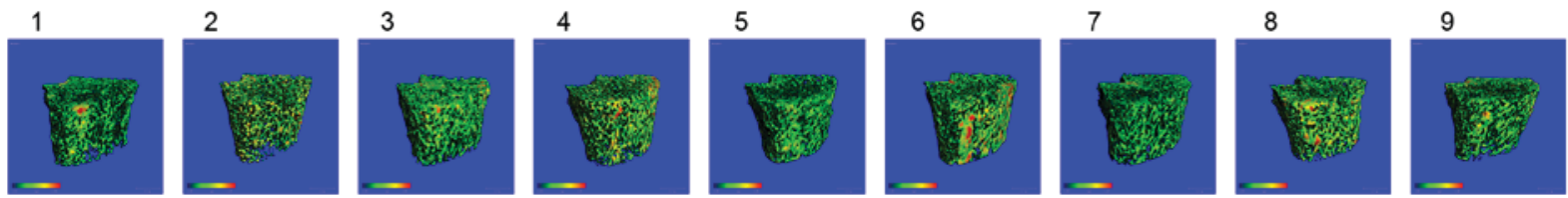

Figure 11. Three-dimensional reconstructed images of the right femur in female rats. (A) SEG image, (B) trabecular SP and (C) trabecular TH. Groups: 1, control; 2, Pred (6 mg/kg/day); 3, Pred + calcitriol ( $0.045 \mu \mathrm{g} / \mathrm{kg} / \mathrm{day}) ; 4$, Pred $+30 \%$ ethanolic eluate of PM (high dose; $400 \mathrm{mg} / \mathrm{kg} / \mathrm{day}) ; 5$, Pred $+30 \%$ ethanolic eluate of PM (medium dose; $200 \mathrm{mg} / \mathrm{kg} / \mathrm{day}$ ); 6, Pred $+30 \%$ ethanolic eluate of PM (low dose; $100 \mathrm{mg} / \mathrm{kg} / \mathrm{day}$ ); 7, Pred + crude extract of PM $(\mathrm{H} ; 400 \mathrm{mg} / \mathrm{kg} / \mathrm{d}) ; 8$, Pred + crude extract of PM (M; $200 \mathrm{mg} / \mathrm{kg} /$ day); 9, Pred + crude extract of PM (L; $100 \mathrm{mg} / \mathrm{kg} /$ day). For SP images, green indicates areas of high bone density and red indicates areas of low bone density. For TH images, green indicates areas of low bone density and red indicates areas of high bone density. Magnification, x30. SEG, segmentation; SP, separation; Th, thickness; Pred, prednisone; PM, Polygonum multiflorum.

52 and $27.0 \%$ in the femur and $\mathrm{LV}_{4}$, respectively, of female rats compared with that in the prednisone groups. In the present study, cross-sectional images from micro-CT scans and longitudinal analysis of the distal femur excluding the cortical bone were performed in the measurement area between 1 and $4 \mathrm{~mm}$ distal to the growth plate-metaphysis junction $(29,30)$, as a previous study had indicated that trabecular bone is more responsive to pharmacological intervention than cortical bone (3).

In conclusion, rats exposed to prednisone showed abnormal bone microstructure as well as decreased BMD and biomechanical properties accompanied with inhibition of skeletal muscle and immune organs. It was demonstrated that 

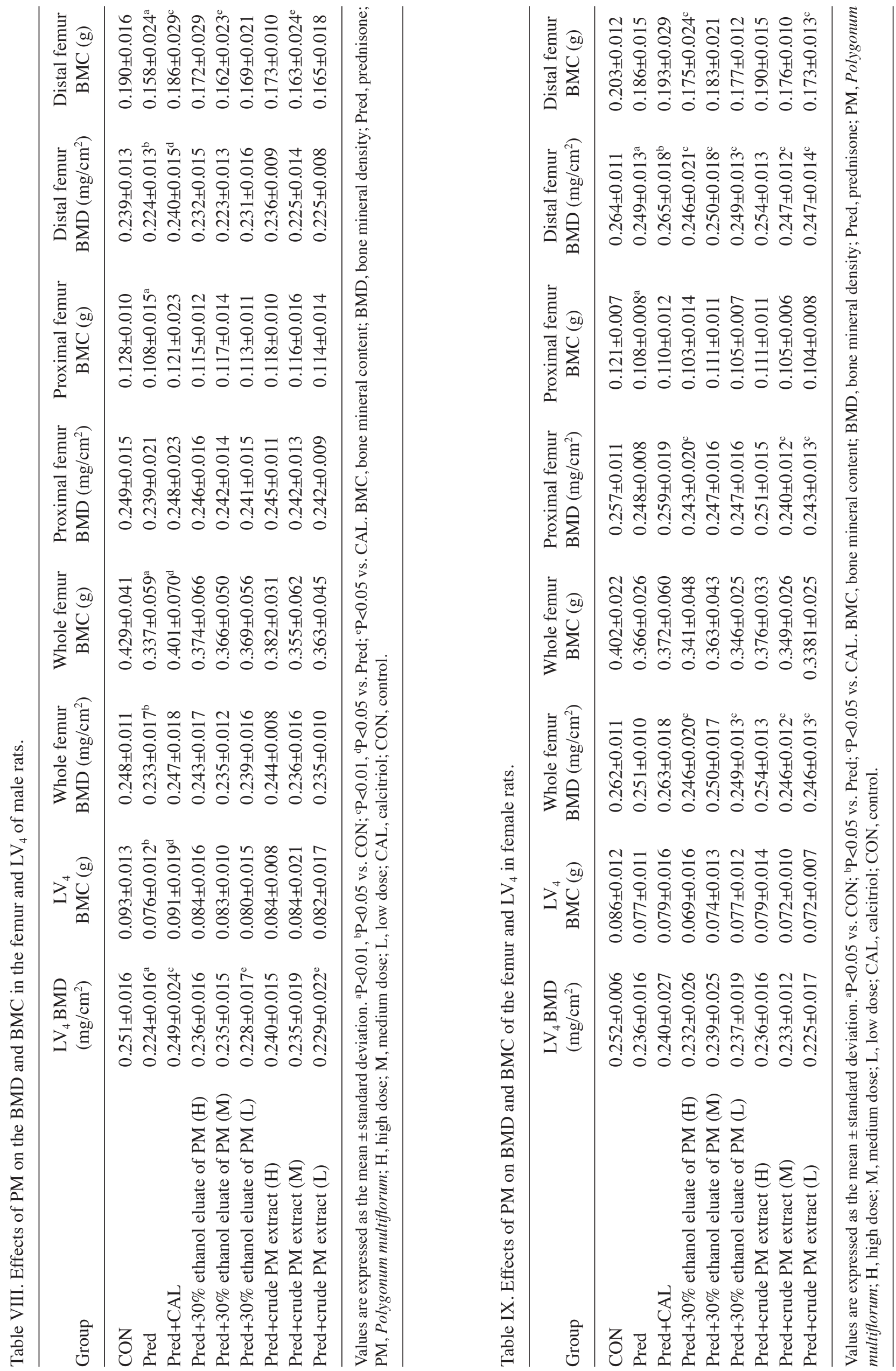

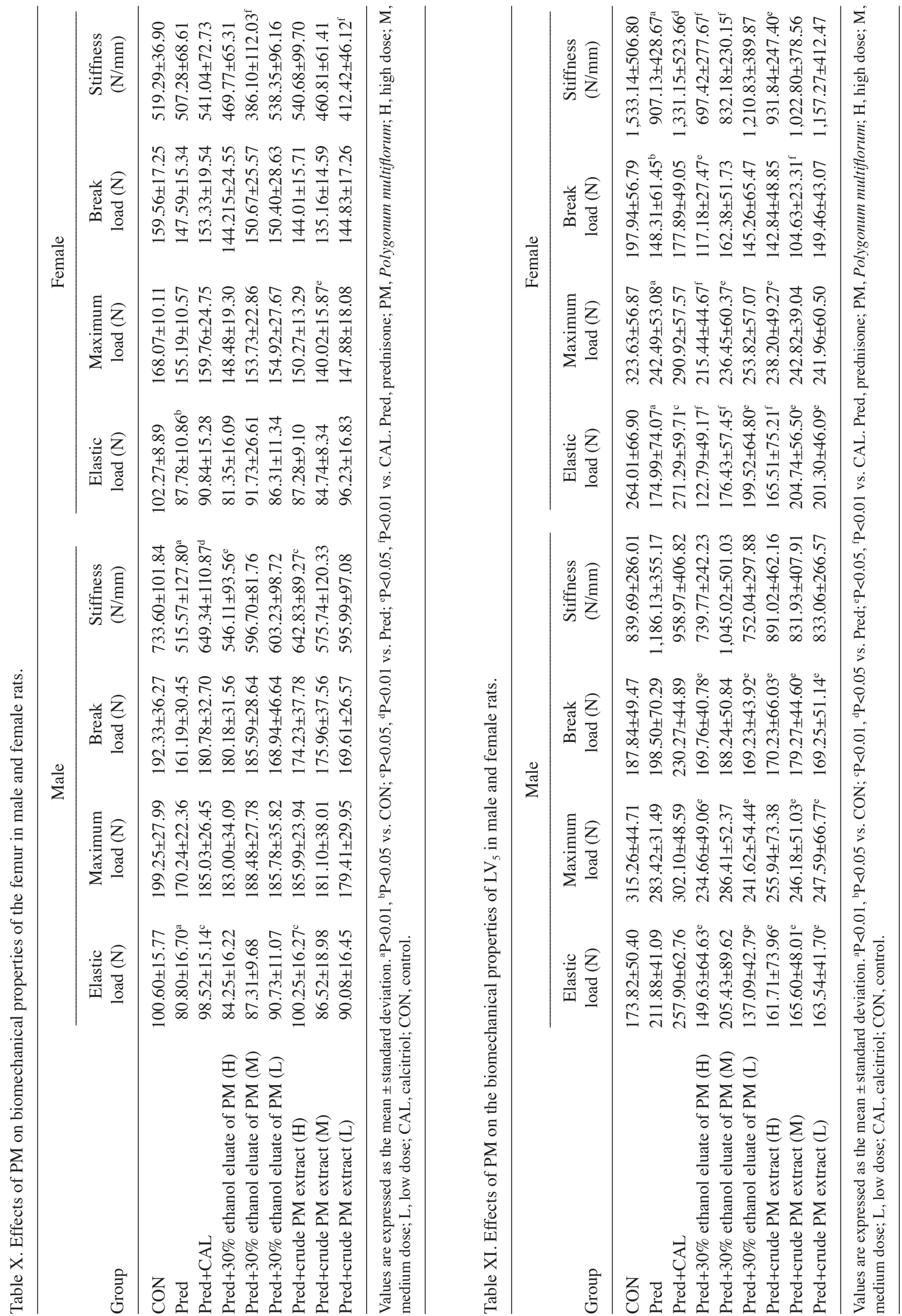
A SEG
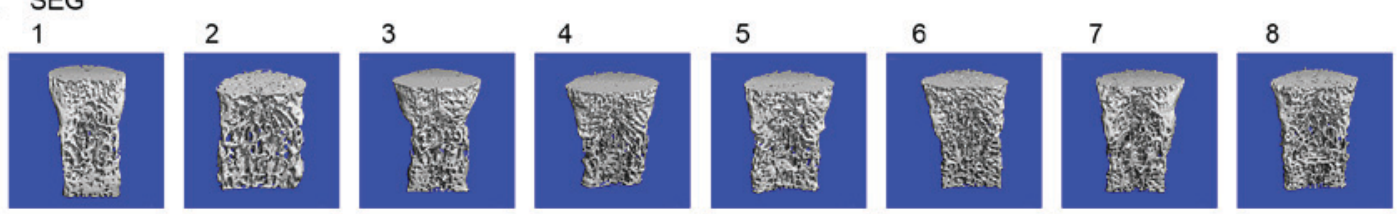

9

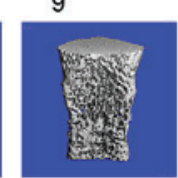

B SP
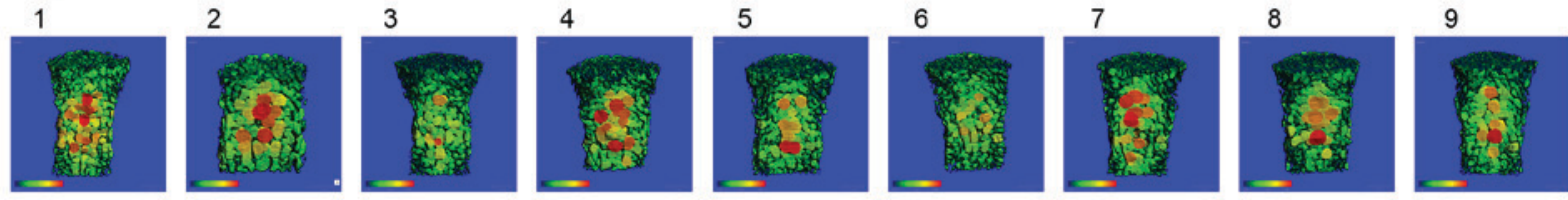

C $\mathrm{TH}$
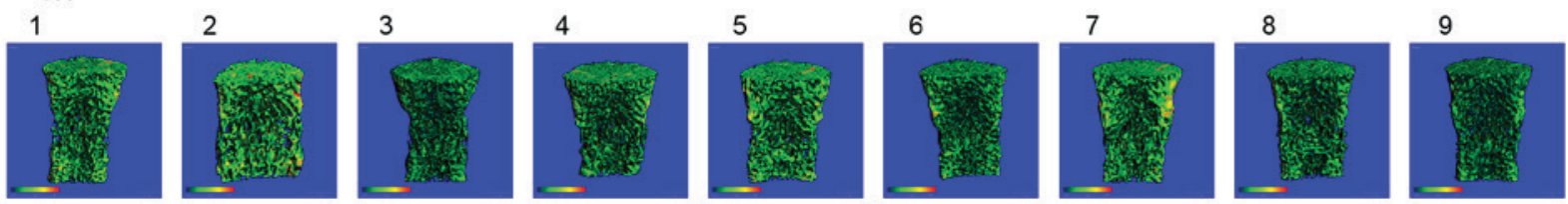

Figure 12. Three-dimensional reconstructed images of the $\mathrm{LV}_{4}$ in male rats. (A) SEG image, (B) trabecular SP and (C) trabecular TH. Groups: 1, control; 2 , Pred (6 mg/kg/day); 3, Pred + calcitriol (0.045 $\mu \mathrm{g} / \mathrm{kg} /$ day); 4, Pred $+30 \%$ ethanolic eluate of PM (high dose; $400 \mathrm{mg} / \mathrm{kg} / \mathrm{day}) ; 5$, Pred + 30\% ethanolic eluate of PM (medium dose; $200 \mathrm{mg} / \mathrm{kg} /$ day); 6, Pred + 30\% ethanolic eluate of PM (low dose; $100 \mathrm{mg} / \mathrm{kg} /$ day); 7, Pred + crude extract of PM (H; 400 mg/kg/d); 8, Pred + crude extract of PM (M; $200 \mathrm{mg} / \mathrm{kg} / \mathrm{day}) ; 9$, Pred + crude extract of PM (L; $100 \mathrm{mg} / \mathrm{kg} /$ day). For SP images, green indicates areas of high bone density and red indicates areas of low bone density. For TH images, green indicates areas of low bone density and red indicates areas of high bone density. Magnification, x30. SEG, segmentation; SP, separation; Th, thickness; Pred, prednisone; PM, Polygonum multiflorum.

A SEG
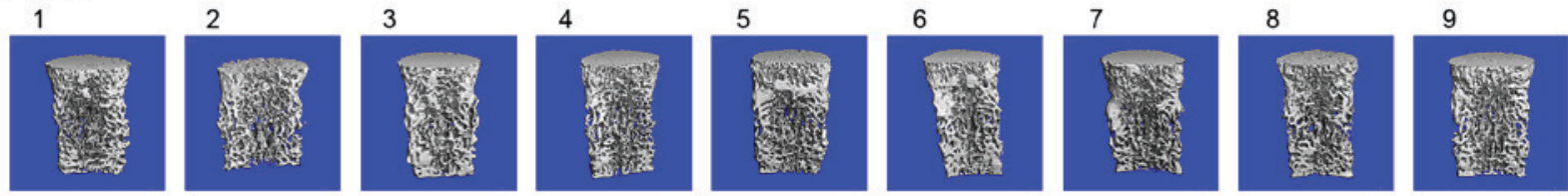

B SP
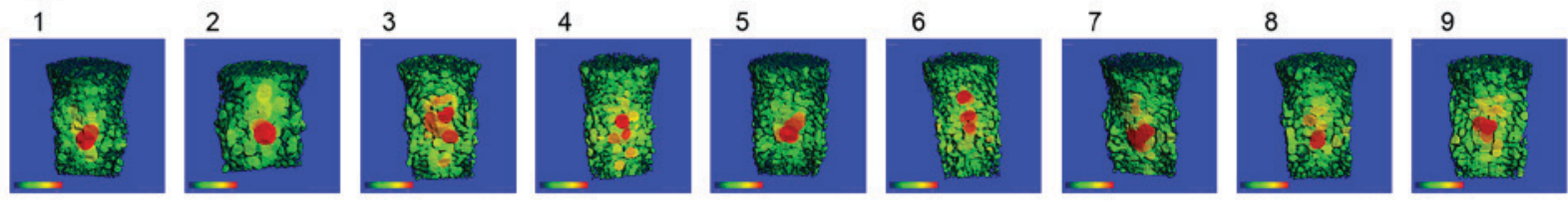

C TH
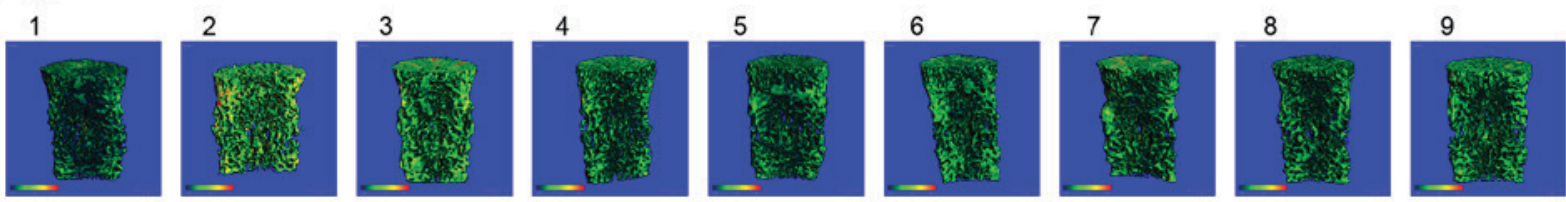

Figure 13. Three-dimensional reconstructed images of the $\mathrm{LV}_{4}$ in female rats. (A) SEG image, (B) trabecular SP and (C) trabecular TH. Groups: 1, control; 2 , Pred (6 mg/kg/day); 3, Pred + calcitriol ( $0.045 \mu \mathrm{g} / \mathrm{kg} / \mathrm{day}) ; 4$, Pred $+30 \%$ ethanolic eluate of PM (high dose; $400 \mathrm{mg} / \mathrm{kg} / \mathrm{day}) ; 5$, Pred $+30 \%$ ethanolic eluate of PM (medium dose; $200 \mathrm{mg} / \mathrm{kg} / \mathrm{day}) ; 6$, Pred $+30 \%$ ethanolic eluate of PM (low dose; $100 \mathrm{mg} / \mathrm{kg} / \mathrm{day}) ; 7$, Pred + crude extract of PM (H; $400 \mathrm{mg} / \mathrm{kg} / \mathrm{d}$ ); 8, Pred + crude extract of PM (M; $200 \mathrm{mg} / \mathrm{kg} /$ day); 9, Pred + crude extract of PM (L; $100 \mathrm{mg} / \mathrm{kg} /$ day). For SP images, green indicates areas of high bone density and red indicates areas of low bone density. For TH images, green indicates areas of low bone density and red indicates areas of high bone density. Magnification, x30. SEG, segmentation; SP, separation; Th, thickness; Pred, prednisone; PM, Polygonum multiflorum.

$30 \%$ ethanolic eluate of PM (M, L) and crude extract of PM (H) exerted a preventive effect on bone loss in rats treated by prednisone. Further investigations are desirable to confirm the mechanism of bone loss prevention by PM in vivo. The present study provided basic evidence for developing PM as a therapeutic medication for the prevention and/or treatment of GIO.

\section{Acknowledgements}

This research was supported by grants from the National Natural Science Foundation of China (no. 81102450 and 81673814), the Open Fund Project of Key Laboratory of Guangdong Province (no. 4CX16010 G), the Science and Technology 
Plan of Guangdong Province (no. 2016ZC0178) and the Characteristic Innovation Project (Natural Science) of the Education Department of Guangdong Province (no. 2014KTSCX084).

\section{References}

1. Mazziotti G, Angeli A, Bilezikian JP, Canalis E and Giustina A: Glucocorticoid-induced osteoporosis: An update. Trends Endocrinol Metab 17: 144-149, 2006.

2. Xia X, Kar R, Gluhak-Heinrich J, Yao W, Lane NE, Bonewald LF, Biswas SK, Lo WK and Jiang JX: Glucocorticoid-induced autophagy in osteocytes. J Bone Miner Res 11: 2479-2488, 2010.

3. Derakhshanian H, Djalali M, Djazayery A, Nourijelyani K, Ghadbeigi S, Pishva H, Saedisomeolia A, Bahremand A and Dehpour AR: Quercetin prevents experimental glucocorticoid-induced osteoporosis: A comparative study with alendronate. Can J Physiol Pharmacol 91: 380-385, 2013.

4. Cheng Y, Wang WL and Liang JJ: Genistein attenuates glucocorticoid-induced bone deleterious effects through regulation Eph/ephrin expression in aged mice. Int J Clin Exp Pathol 8: 394-403, 2015.

5. Plotkin LI, Bivi N and Bellido T: A bisphosphonate that does not affect osteoclasts prevents osteoblast and osteocyte apoptosis and the loss of bone strength induced by glucocorticoids in mice. Bone 49: 122-127, 2011.

6. Kalak R, Zhou H, Street J, Day RE, Modzelewski JR, Spies CM, Liu PY, Li G, Dunstan CR and Seibel MJ: Endogenous glucocorticoid signalling in osteoblasts is necessary to maintain normal bone structure in mice. Bone 45: 61-67, 2009.

7. Canalis E: Mechanisms of glucocorticoid action in bone. Curr Osteoporos Rep 3: 98-102, 2005.

8. Gennari L and Bilezikian JP: Glucocorticoid-induced osteoporosis: Hope on the HORIZON. Lancet 373: 1253-1256, 2009.

9. Barnes PJ: Glucocorticosteroids: Current and future directions. Br J Pharmacol 163: 29-43, 2011.

10. Weinstein RS: Glucocorticoid-induced osteoporosis and osteonecrosis. Endocrinol Metab Clin North Am 41: 595-611, 2012.

11. Tory HO, Solomon DH and Desai SP: Analysis of quality improvement efforts in preventing glucocorticoid-induced osteoporosis. Semin Arthritis Rheum 44: 483-488, 2009.

12. Lekamwasam S, Adachi JD, Agnusdei D, Bilezikian J, Boonen S, Borgström F, Cooper C, Diez Perez A, Eastell R, Hofbauer LC, et al: A framework for the development of guidelines for the management of glucocorticoid-induced osteoporosis. Osteoporos Int 23: 2257-2276, 2012.

13. Mazzantini M and Di Munno O: Glucocorticoid-induced osteoporosis: 2013 update. Reumatismo 66: 144-152, 2014.

14. Chung HJ, Kyung Kim W, Joo Park H, Cho L, Kim MR, Kim MJ, Shin JS, Ho Lee J, Ha IH and Kook Lee S: Anti-osteoporotic activity of harpagide by regulation of bone formation in osteoblast cell culture and ovariectomy-induced bone loss mouse models. J Ethnopharmacol 179: 66-75, 2016.

15. Jeon YK, Kim KM, Kim KJ, Kim IJ, Lim SK and Rhee Y: The anabolic effect of teriparatide is undermined by low levels of high-density lipoprotein cholesterol. Calcif Tissue Int 94 159-168, 2014.

16. Ngueguim FT, Khan MP, Donfack JH, Siddiqui JA, Tewari D, Nagar GK, Tiwari SC, Theophile D, Maurya R and Chattopadhyay N: Evaluation of Cameroonian plants towards experimental bone regeneration. J Ethnopharmacol 141: 331-337, 2012.

17. Srivastava K, Tyagi AM, Khan K, Dixit M, Lahiri S, Kumar A, Changkija B, Khan MP, Nagar GK, Yadav DK, et al: Isoformononetin, a methoxydaidzein present in medicinal plants, reverses bone loss in osteopenic rats and exerts bone anabolic action by preventing osteoblast apoptosis. Phytomedicine 20: 470-480, 2013.

18. Gao Z, Lu Y, Halmurat U, Jing J and Xu D: Study of osteoporosis treatment principles used historically by ancient physicians in chinese medicine. Chin J Integr Med 19: 862-868, 2013.

19. Huang LF, Wu T, Xie H and Liao JM: The prevention and treatment on bone loss in ovariectomized rats of Polygonum decoction. Chin J Gerontol 25: 709-710, 2005 (In Chinese).

20. Wu Tie, Cui Liao, Li Qingnan, Xie Hua, Zhang Zhiping, Chen Zhidong and Fu Yuping: The new use of Polygonum Multiflorum and its extracts on prevention and treatment of osteoporosis. Patent no. ZL 001 01246.0. Filed January 22,2000; issued July 16, 2003.

21. Liu YY, Cui L, Wu T and Yao WM: Effects of emodin on the proliferation and differentiation of osteoblast isolated from neonatal rat calvarium in vitro. Chin Pharmacol Bull 21: 235-240, 2005 (In Chinese).
22. Liu YY, Cui L, Wu T and Yao WM: Effects of emodin on adipogenesis of marrow stromal cells in vitro. Chin Pharmacol Bull 21: 842-846, 2005 (In Chinese).

23. Liu YY, Yao WM, Ai CM, Xu BL, Zou LY, Cui L and Wu T: Effects of emodin on differentiation of bone marrow stroma cell into osteoblast in rats in vitro. Chin J Clin Pharmacol Ther 10: 191-195, 2005 (In Chinese).

24. Liu YY, Yao WM, Ai CM, Xu BL, Zou LY, Wu T and Cui L: Effects of emodin on osteoblast in vitro. Chin Pharmacol Bull 21: 1473-1477, 2005 (In Chinese).

25. Liang L, Zhao Z and Kang T: Application of microscopy technique and high performance liquid chromatography for quality assessment of Polygonum multiflorum Thunb (Heshouwu). Pharmacogn Mag 10: 415-421, 2014.

26. Chappard D, Baslé MF, Legrand E and Audran M: New laboratory tools in the assessment of bone quality. Osteoporos Int 22: 2225-2240, 2011

27. Kliwinski C, Kukral D, Postelnek J, Krishnan B, Killar L, Lewin A, Nadler S and Townsend R: Prophylactic administration of abatacept prevents disease and bone destruction in a rat model of collagen-induced arthritis. J Autoimmun 25: 165-171, 2005.

28. Liu C, Zhang Y, Kong X, Zhu L, Pang J, Xu Y, Chen W, Zhan H, Lu A and Lin N: Triptolide prevents bone destruction in the collagen-induced arthritis model of rheumatoid arthritis by targeting RANKL/RANK/OPG signal pathway. Evid Based Complement Alternat Med 2013: 626038, 2013.

29. Spies CM, Wiebe E, Tu J, Li A, Gaber T, Huscher D, Seibel MJ, Zhou $\mathrm{H}$ and Buttgereit F: Acute murine antigen-induced arthritis is not affected by disruption of osteoblastic glucocorticoid signalling. BMC Musculoskelet Disord 15: 31, 2014.

30. Wu Q, Xiong X, Zhang X, Lu J, Zhang X, Chen W, Wu T, Cui L, Liu Y and Xu B: Secondary osteoporosis in collagen-induced arthritis rats. J Bone Miner Metab 34: 500-516, 2016.

31. Zeng JH, Zhong ZM, Li XD, Wu Q, Zheng S, Zhou J, Ye WB, Xie F, Wu XH, Huang ZP and Chen JT: Advanced oxidation protein products accelerate bone deterioration in aged rats. Exp Gerontol 50: 64-71, 2014.

32. Cui L, Li T, Liu Y, Zhou L, Li P, Xu B, Huang L, Chen Y, Liu Y, Tian X, et al: Salvianolic acid B prevents bone loss in prednisone-treated rats through stimulation of osteogenesis and bone marrow angiogenesis. PLoS One 7: e34647, 2012.

33. Khana MP, Mishrab JS, Sharana K, Yadavb M, Singhb AK, Srivastavac A, Kumar S, Bhaduaria S, Maurya R, Sanyal S and Chattopadhyay N: A novel flavonoid C-glucoside from Ulmus wallichiana preserves bonemineral density, microarchitecture and biomechanical properties in the presence of glucocorticoid by promoting osteoblast survival: A comparative study with human parathyroid hormone. Phytomedicine 20: 1256-1266, 2013.

34. Palombarini M, Gombia M, Fantazzini P, Giardino R, Giavaresi G, Parrilli A, Vittur F and Guillot G: Inhomogeneity of rat vertebrae trabecular architecture by high-field 3D mu-magnetic resonance imaging and variable threshold image segmentation. J Magn Reson Imaging 30: 825-833, 2009.

35. Ilias I and Ghayee H: Glucocorticoid-induced osteoporosis. De Groot LJ, Chrousos G, Dungan K, Feingold KR, Grossman A, Hershman JM, Koch C, Korbonits M, McLachlan R, New M, Purnell J, Rebar R, Singer F, Vinik A (eds). Source: Endotext [Internet], MDText.com <[http://MDText.com/]>, Inc., South Dartmouth, MA, Jan 7, 2015.

36. Canalis E: Mechanisms of glucocorticoid-induced osteoporosis. Curr Opin Rheumatol 15: 454-457, 2003.

37. Arango-Lievano M, Bodine SC and Cato CB: Glucocorticoid signaling from molecules to mice to man. Advances In Experimental Medicine and Biology 872: 179-216, 2015.

38. Pereira RM, Delany AM and Canalis E: Cortisol inhibits the differentiation and apoptosis of osteoblasts in culture. Bone 28: 484-490, 2001

39. Zhou MR, Li J, Wu JK, Zeng XB, Chen JF, Cui L, and Liu YY: The preventive effect of Polygonum multiflorum on the changes of micro-structural and biomechanical by prednisone. Chin Pharmacol Bull 31: 1273-1279, 2015 (In Chinese).

40. Zheng YY: Seperation and purification of the Polygonum multiflorum extract and its effect on anti-osteoporosis. Guangdong Med Univ, 2014 (In Chinese).

41. Zhu FP, Wang HF and Gao LF: Effects of kidney-nourishing herbs on renal 1- $\alpha$-hydroxylase. Shanghai J of Trad Chin Med 37: 42, 2003 (In Chinese). 